TRANSACTIONS OF THE

AMERICAN MATHEMATICAL SOCIETY

Volume 2X6. Number 2. December 1984

\title{
MINIMAL PERIODIC ORBITS FOR CONTINOUS MAPS OF THE INTERVAL
}

\author{
BY
}

LLUIS ALSEDA, JAUME LLIBRE AND RAFEL SERRA

\begin{abstract}
For continuous maps of the interval into itself, Sarkovskii's Theorem gives the notion of minimal periodic orbit. We complete the characterization of the behavior of minimal periodic orbits. Also, we show for unimodal maps that the min-max essentially describes the behavior of minimal periodic orbits.
\end{abstract}

1. Introduction. We say that a periodic orbit of period $n$ of a continuous map $f$ of the interval is minimal, if $\boldsymbol{n}$ is the minimal period of $f$ in Sarkovskii's ordering (see Definition 2.2). The aim of this paper is to characterize the behavior of a minimal periodic orbit relative to its natural ordering as a finite subset of the real line.

A periodic orbit $P$ of a continuous map $f$ of the interval will be called simple if $f$ has a particular behavior on $P$, given in Definitions 2.3, 2.8, 2.15 and Proposition 4.6. The definitions of simple periodic orbit of period odd and a power of two were given by Stefan and Block, respectively. Also, for the above two cases they proved that a minimal periodic orbit has simple behavior.

The main result of this paper is to complete the characterization of the behavior of the minimal periodic orbits of continuous mappings of the interval. In fact we prove that every minimal periodic orbit has simple behavior. Moreover, for each simple behavior, that is for each simple periodic orbit $P$, we show there is a continuous map of the interval having $P$ as a minimal periodic orbit (see Theorem 2.17 and Propositions 2.7 and 2.13).

For unimodal maps it is known that there is a strong relation between Sarkovskii's ordering and the min-max (see Theorem II.2.8 of [CE]). So, for unimodal maps, the notions of minimal periodic orbit and min-max are related to Sarkovskii's ordering. This implies the existence of some relation between them. The purpose of this paper on unimodal maps is to show the equivalence between the behavior described by simple periodic orbits and the "min-max itinerary" (see Theorem 3.4). Moreover, we prove that if an unimodal map has period $n$, then it has (at least) one simple periodic orbit of period $n$ (see Theorem 3.5). Also, in Proposition 5.8, we characterize the shape of simple periodic orbits restricted to unimodal maps.

Theorem 3.4 was presented without proof in [AS].

Received by the editors May 12, 1983 and, in revised form, December 12, 1983.

1980 Mathematics Subject Classification. Primary 54H20.

Key words and phrases. Periodic point, minimal periodic orbit, simple periodic orbit, unimodal map, $\min -\max$.

(C)1984 American Mathematical Society $0002-9947 / 84 \$ 1.00+\$ .25$ per page 
2. Minimality and simple periodic orbits. Statement of results. Let $I$ denote a closed interval on the real line and $C(I)$ the set of continuous maps from $I$ to itself. A point $p \in I$ is a periodic point of a map $f \in C(I)$ if $f^{n}(p)=p$ for some positive integer $n$. The period of $p$ is the least such integer $n$, and the orbit of $p$ is the set $P=\operatorname{Orb}(p)=\left\{f^{k}(p): k=1,2, \ldots, n\right\}$. We refer to such an orbit as a periodic orbit of period $n$. Let $P(f)$ denote the set of positive integers $n$ such that $f$ has a periodic orbit of period $n$.



(a)

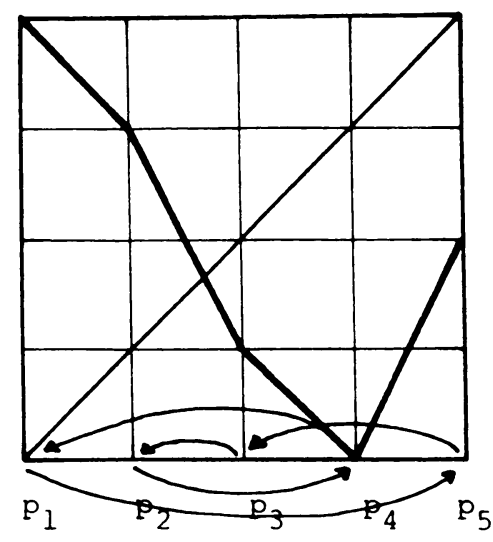

(b)

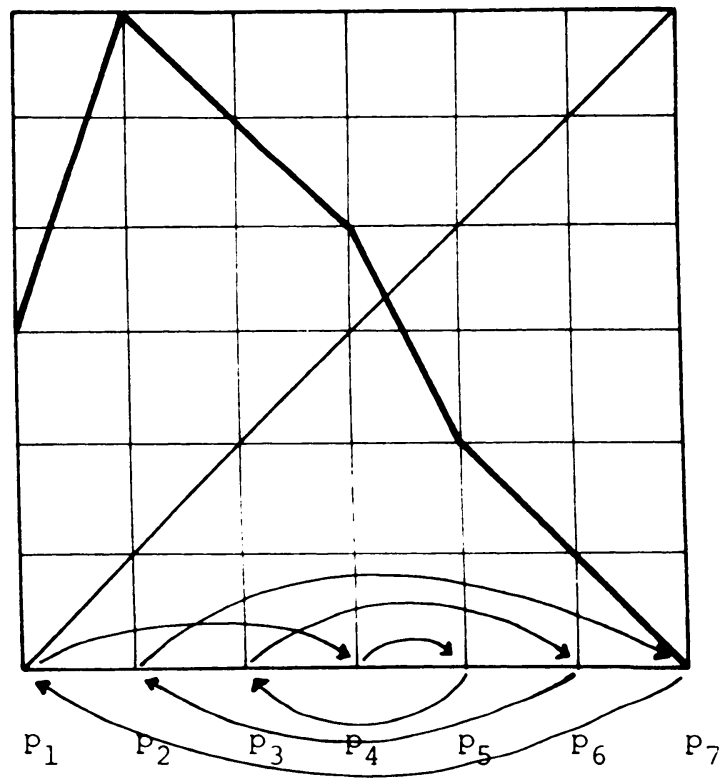

(c)

Figure 1. Figures 1(a) and (c) show the shape and the graph of a map, on a SPO ${ }^{+}$of period 5 and 7. respectively. Figure $1(\mathrm{~b})$ shows the same for a $\mathrm{SPO}^{-}$of period 5 . 

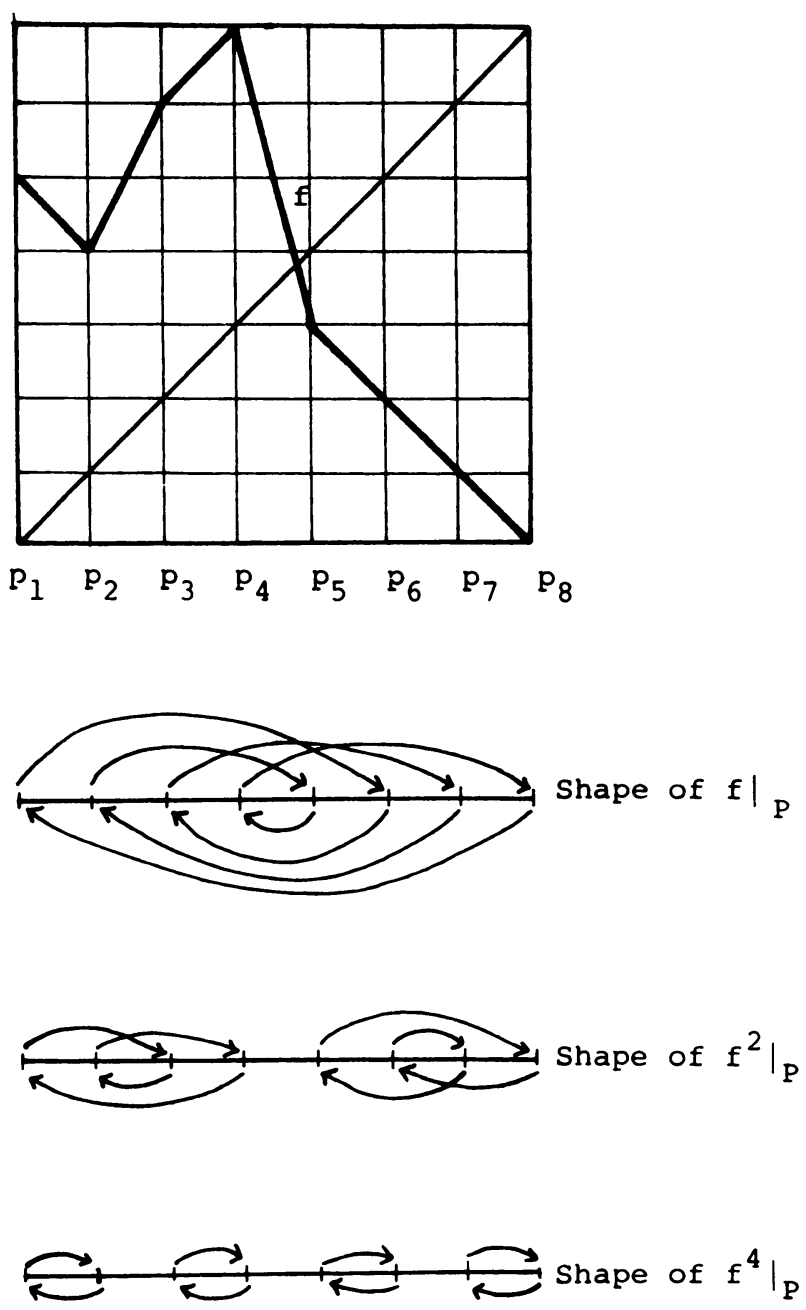

FigURE 2. This figure shows the graph of a map $f$ which has a SPO $P=\left\{p_{1}, p_{2}, \ldots, p_{k}\right\}$ and the shape of $f$ on $P$.

Let $N$ denote the set of positive integers and $\rightarrow$ denote the following ordering of $N$ :

$$
\begin{aligned}
3 & \rightarrow 5 \rightarrow 7 \rightarrow \cdots \rightarrow 2.3 \rightarrow 2.5 \rightarrow 2.7 \rightarrow \cdots \\
& \rightarrow 4.3 \rightarrow 4.5 \rightarrow 4.7 \rightarrow \cdots \rightarrow 8 \rightarrow 4 \rightarrow 2 \rightarrow 1 .
\end{aligned}
$$

In the $\rightarrow$ ordering, called Sarkovskii's ordering, the smallest element is 3 and the greatest element is 1 .

TheOREM 2.1 (SARKovSKiI [Sa, St, BGMY]). Let $f \in C(I)$ and suppose $n \in P(f)$ and $n \rightarrow k$. Then $k \in P(f)$.

Definition 2.2. Let $f \in C(I)$. Suppose $P(f) \neq\{1,2,4,8,16, \ldots\}$ and let $n>1$ be the smallest element of $P(f)$ in the $\rightarrow$ ordering. We say that a periodic orbit is 




Figure 3 
minimal if its period is $n$, and we refer to such an orbit as MPO. Note that if $P(f)=\{1,2,4,8,16, \ldots\}$, then the smallest element of $P(f)$ in the $\rightarrow$ ordering does not exist.

Definition 2.3. Let $P=\left\{p_{1}, p_{2}, \ldots, p_{n}\right\}$ be a periodic orbit of $f \in C(I)$, with $p_{1}<p_{2}<\cdots<p_{n}$, of period $n=2^{m} q$ where either $m \geqslant 0$ and $q=1$ or $m=0$ and $q \geqslant 3$. Suppose $m=0$ and let $t=(q+1) / 2$. We say that $P$ is a simple periodic orbit of type + or, equivalently, $\mathrm{SPO}^{+}$, if

$$
\begin{array}{ll}
f\left(p_{t-k}\right)=p_{t+k+1} & \text { for } k=0,1,2, \ldots, t-2, \\
f\left(p_{t+k}\right)=p_{t-k} & \text { for } k=1,2,3, \ldots, t-1, \text { and } \\
f\left(p_{1}\right)=p_{t} . &
\end{array}
$$

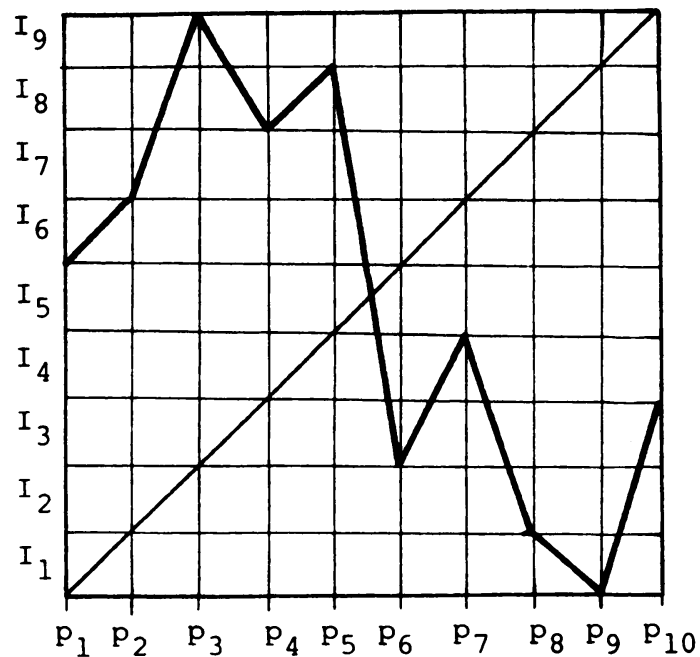

FIGURE 4

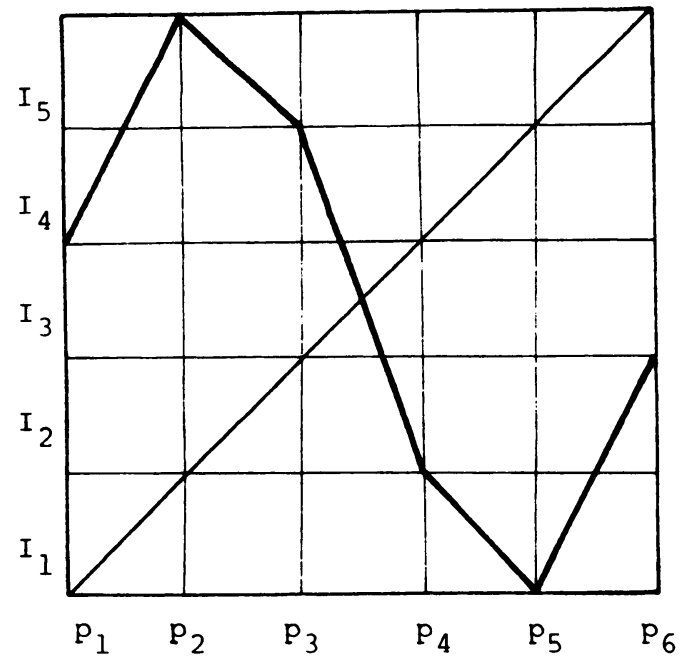

Figure 5 
Similarly we say that $P$ is a simple periodic orbit of type - or, equivalently, $\mathrm{SPO}^{-}$, if

$$
\begin{array}{ll}
f\left(p_{t-k}\right)=p_{t+k} & \text { for } k=1,2,3, \ldots, t-1, \\
f\left(p_{t+k}\right)=p_{t-k-1} & \text { for } k=0,1,2, \ldots, t-2, \text { and } \\
f\left(p_{q}\right)=p_{t} . &
\end{array}
$$

For the case $q=1$ we define a simple periodic orbit, SPO, inductively. If $m=1$ then $P$ is simple. Suppose $m>1$. Then we say $P$ is simple if the two subsets $\left\{p_{1}, p_{2}, \ldots, p_{n / 2}\right\}$ and $\left\{p_{(n / 2)+1}, \ldots, p_{n}\right\}$ of $P$ are simple periodic orbits of period $n / 2$ of $f^{2}$. Then we have $f\left(\left\{p_{1}, p_{2}, \ldots, p_{n / 2}\right\}\right)=\left\{p_{(n / 2)+1}, \ldots, p_{n}\right\}$ (see Figures 1 and 2).

This definition was given by Stefan [St] and Block [B|1] for the cases $m=0$ and $q=1$, respectively.

Remark 2.4. Let $P_{1}$ and $P_{2}$ be two finite totally ordered sets and let $f_{1}: P_{1} \rightarrow P_{1}$ and $f_{2}: P_{2} \rightarrow P_{2}$ be two bijective maps. We say that $f_{1}$ and $f_{2}$ are isomorphic if there exists a bijective map $g: P_{1} \rightarrow P_{2}$ such that $g \circ f_{1}=f_{2} \circ g$ and $g$ is either order preserving or order reversing. Let $a_{m}$ be the number of nonisomorphic simple periodic orbits of period $2^{m}$. Then we have that $a_{m}=2^{2^{m}-m-1}$.

The computation of $a_{m}$ (see $\$ 4$ ) gives us, in particular, an algorithm to construct the SPO of period $2^{m}$.

Definition 2.5. Let $f \in C(I)$ and let $P=\left\{p_{1}, p_{2}, \ldots, p_{n}\right\}$ be a periodic orbit of $f$ where $p_{1}<p_{2}<\cdots<p_{n}$. We denote by $\bar{f}$ the map such that:

(1) $\bar{f} \in C(I)$,

(2) $\bar{f}(x)=f\left(p_{1}\right)$ for $x \leqslant p_{1}$,

(3) $\bar{f}(x)=f\left(p_{n}\right)$ for $x \geqslant p_{n}$,

(4) $\bar{f}\left(p_{i}\right)=f\left(p_{i}\right)$ for $i=1,2, \ldots, n$,

(5) $\left.\bar{f}\right|_{\left[p_{i}, p_{i+1}\right]}$ is linear for $i=1,2, \ldots, n-1$.

We call $\bar{f}$ the $P$-linearization of $f$. If $\bar{f}=f$ we say that $f$ is $P$-linear.

Then we have the following results.

THEOREM 2.6. Let $f \in C(I)$ and suppose $P$ is a periodic orbit of $f$ of period $n$. Then the following statements hold.

(1) (Theorem 2 of [St]) Suppose $n \geqslant 3$ odd and $P$ is minimal; then $P$ is simple.

(2) (See [St].) Suppose $P$ is simple, $n \geqslant 3$ odd, and $f$ is $P$-linear; then $P$ is the unique minimal periodic orbit of $f$.

(3) (Theorem A of [B11]) $f$ has no periodic points with period different from a power of two if and only if every periodic orbit of $f$ with period a power of two is simple. In particular, if $P$ is minimal and $n=2^{m}$ with $m \geqslant 0$, then $P$ is simple.

Statement (2) is a kind of "converse" of statement (1). In a similar way we give, in the next proposition, the "converse" of statement (3).

Proposition 2.7. Let $f \in C(I)$ and suppose $P$ is a simple periodic orbit of $f$ with period $n=2^{m}$ where $m \geqslant 0$. If, furthermore, $f$ is $P$-linear, then $P$ is the unique minimal periodic orbit of $f$. 
These results state the "equivalence" between the notions of MPO and SPO with period odd (different from 1) or power of two. So, for these periods, the above equivalence characterizes the behavior of a MPO. On the other hand, we know that if $f \in C(I)$ and $P(f)=\left\{2^{m}: m \geqslant 0\right\}$, then the MPO does not exist. However, Theorem 2.6(3) gives us all the information in terms of the behavior of $f$ on their periodic orbits.

We prove Proposition 2.7 in $\$ 4$. To do this we use a result from [UR] which classified the $A$-graph of a SPO of period $2^{m}, m \geqslant 1$.

If we look for a similar result to Theorem 2.6 and Proposition 2.7 for a periodic orbit of period $n=2^{m} q$ with $m \geqslant 1$ and $q \geqslant 3$ odd, it seems to be natural to define SPO similarly to the case $n=2^{m}$.

Definition 2.8. Let $P$ be a periodic orbit of $f \in C(I)$ of period $n=2^{m} q$ with $m \geqslant 1$ and $q \geqslant 3$ odd. We define a weak simple periodic orbit, WSPO, inductively. When $m=1, P$ is a WSPO if the subsets $\left\{p_{1}, p_{2}, \ldots, p_{q}\right\}$ and $\left\{p_{q+1}, p_{q+2}, \ldots, p_{2 q}\right\}$ are SPO of $f^{2}$ of period $q$. When $m>1, P$ is a WSPO if the subsets $\left\{p_{1}, p_{2}, \ldots, p_{n / 2}\right\}$ and $\left\{p_{(n / 2)+1}, \ldots, p_{n}\right\}$ of $P$ are WSPO of $f^{2}$ of period $n / 2$. See Figures 3,4 and 5 for examples with $n=20,10$ and 6, respectively.

Definition 2.9. Let $P$ be a periodic orbit of $f \in C(I)$. We set $Q_{i}=\left\{p_{j} \in P\right.$ : $(i-1) q+1 \leqslant j \leqslant i q\}$ for $i=1,2, \ldots, w$, where $w=2^{m}$. Moreover, we denote by $p_{j}^{i}$ the point $p_{k} \in P$ with $k=(i-1) q+j$, where $i=1,2, \ldots, w$ and $j=1,2, \ldots, q$. That is, $Q_{i}=\left\{p_{1}^{i}, p_{2}^{i}, \ldots, p_{q}^{i}\right\}$ with $p_{1}^{i}<p_{2}^{i}<\cdots<p_{q}^{i}$ and $\left(p_{j}^{i}, p_{j+1}^{i}\right) \cap P=\varnothing$ for $j=1,2, \ldots, q-1$. In a similar way we define $G_{i}=\left[p_{1}^{i}, p_{q}^{i}\right]$ and $I_{j}^{i}=\left[p_{j}^{i}, p_{j+1}^{i}\right]$ for $i=1,2, \ldots, w$ and $j=1,2, \ldots, q-1$ (see Figure 3).

Definition 2.10. Let $B$ be a finite set. We say $B$ is a periodic set of period $n$ if $f^{n}(B)=B$ and $f^{k}(B) \cap B=\varnothing$ for $k=1,2, \ldots, n-1$. The orbit of $B$ is the set $\left\{f^{k}(B): k=1,2, \ldots, n\right\}$. Let $B$ be a periodic set and $O_{B}=\left\{B_{1}, B_{2}, \ldots, B_{n}\right\}$ its orbit. Suppose $O_{B}$ is labeled such that for every $x \in B_{i}$ and $y \in B_{j}$ we have $x<y$ if and only if $i<j$. Suppose $n=2^{m}$ with $m \geqslant 1$ and $|B|$ is odd or a power of two (where $|B|$ denotes the cardinal of $B$ ). Then we define a simple set inductively. When $m=1, O_{B}$ is a simple set if $B_{1}$ and $B_{2}$ are SPO of $f^{2}$ with period $|B|$. If $m>1$ we say $O_{B}$ is a simple set if the subsets $\left\{B_{1}, B_{2}, \ldots, B_{n / 2}\right\}$ and $\left\{B_{(n / 2)+1}, \ldots, B_{n}\right\}$ are simple sets of $f^{2}$. Note that $f\left(\left\{B_{2}, B_{2}, \ldots, B_{n / 2}\right\}\right)=\left\{B_{(n / 2)+1}, \ldots, B_{n}\right\}$.

In Figure 3 , every set $Q_{i}, i=1,2,3,4$, is a periodic set of period 4 and $\left\{Q_{i}\right.$ : $i=1,2,3,4\}$ is a simple set.

Note that the above definition of simple set is the definition of SPO with period a power of two on finite sets instead of points. Roughly, we can say that a simple set is a "fat simple periodic orbit". In the following remark we give an equivalent definition of WSPO in terms of the sets $Q_{i}$.

REMARK 2.11. Let $P$ be a periodic orbit of $f \in C(I)$ with period $2^{m} q$ where $m \geqslant 1$ and $q \geqslant 3$ odd. Then $P$ is a WSPO if and only if $\left\{Q_{i}: i=1,2, \ldots, w\right\}$ is a simple set (see Figure 3).

REMARK 2.12. Note that, if $P$ is a WSPO of $f$, for the map $f^{2^{m}}$ we have $2^{m}$ SPO of period $q$, and each one is of type + or - . Then if $b_{n}$ is the number of nonisomorphic WSPO of period $n=2^{m} q$ with $m \geqslant 1$ and $q \geqslant 3$ odd, we have $b_{n}=2^{2^{m+1}-m-1}$. $q^{2^{m}-1}$. 
As in Remark 2.4 the computation of $b_{n}$ is given in $\$ 4$ and will give us a way to construct the WSPO.

Proposition 2.13. Let $f \in C(I)$ and suppose $P$ is a MPO of period $2^{m} q$ with $m \geqslant 1$ and $q \geqslant 3$ odd. Then $P$ is a WSPO.

This proposition will be proved in §4. Surprisingly, if we look for the converse of Proposition 2.13 as we did in Theorem 2.6(2) and Proposition 2.7, we find that in this case the converse is not true. Thus, in Example 4.16 (at the end of $\$ 4$ ) we show that the map given in Figure 4 has a WSPO $P$ of period $n=2 \cdot 5$ which is not a MPO. Because of this, to characterize the behavior of a MPO with period $2^{m} q$, we must restrict the definition of WSPO.

DefinItION 2.14. Let $P$ be a WSPO of $f \in C(I)$. We say that $f$ is order preserving on $Q_{i}$, denoted $f \equiv R\left(Q_{i}\right)$, if $f\left(p_{j}^{i}\right)=p_{j}^{k}$ for $j=1,2, \ldots, q$ and $f\left(Q_{i}\right)=Q_{k}$ (here we use Remark 2.11). Similarly we say that $f$ is order reversing on $Q_{i}, f \equiv-R\left(Q_{i}\right)$, if $f\left(p_{j}^{i}\right)=p_{q-j+1}^{k}$ for $j=1,2, \ldots, q$ and $f\left(Q_{i}\right)=Q_{k}$.

Now we can define SPO for the case $2^{m} q, m>0$ and $q \geqslant 3$ odd.

Definition 2.15. Let $P$ be a WSPO of $f \in C(I)$. We say that $P$ is a simple periodic orbit, SPO, if there is an unique $Q_{r}$ such that $f$ is neither order preserving on $Q_{r}$ nor order reversing on $Q_{r}$ (see Figure 8 at the end of $\S 4$ for an example).

In $\$ 4$ (see Proposition 4.6) we give a characterization of a SPO $P$ of a map $f$ in terms of the behavior of $f$ on $P$. In fact, we show that the graph of $f$ on the special $Q_{r}$, such that $f \neq R\left(Q_{r}\right)$ and $f \neq-R\left(Q_{r}\right)$, can be described as in Figure 7 (see $\S 4$ ).

REMARK 2.16. Let $c_{m}$ be the number of nonisomorphic SPO of period $2^{m} q$ with $m \geqslant 1$ and $q \geqslant 3$ odd. Then $c_{m}=2^{2^{m+1}-1}$.

The next theorem is our main result in $\$ 2$.

THEOREM 2.17. Let $P$ be a periodic orbit of $f \in C(I)$ of period $n=2^{m} q$ with $m \geqslant 1$ and $q \geqslant 3$ odd.

(1) If $q>3$ and $P$ is minimal, then $P$ is simple.

(2) If $P$ is simple and $f$ is $P$-linear, then $P$ is the unique minimal periodic orbit of $f$.

(3) If $q=3$ and $P$ is the unique minimal periodic orbit of $f$, then $P$ is simple.

(4) If $q=3, P$ is weak simple and $f$ is $P$-linear, then $P$ is minimal.

Note that Theorem 2.17 extends Theorem 2.6 and Proposition 2.7 to the case $n=2^{m} q$ with $m \geqslant 1$ and $q \geqslant 3$ odd. So, this completes the characterization of the behavior of the minimal periodic orbits.

We note that Theorem 2.17(4) is the "converse" of Proposition 2.13 for the case $q=3$.

The difference between statements (1) and (3) of Theorem 2.17 is the uniqueness of the MPO $P$. To show that the uniqueness is a necessary condition in (3), consider the map $f$ shown in Figure 5. This map has a WSPO $P$ which is not a SPO. In $\$ 4$ (see Example 4.17) we show that $P$ is a MPO of $f$ and that $P$ is not the unique MPO of $f$.

The computation of $c_{m}$ and the proof of Theorem 2.17 will be given in $\$ 4$. The computation of $c_{m}$ will give us (as the computations of $a_{m}$ and $b_{n}$ ) an algorithm to construct all the SPO of period $2^{m} q, m \geqslant 1$ and $q \geqslant 3$ odd. 
In $\S 4$ we also characterize (see Proposition 4.15) the $A$-graph of a map $f$ which has a SPO of period $2^{m} q$ with $m \geqslant 1$ and $q \geqslant 3$ odd. Similar results are given in [BGMY AND LIR] for the cases $m=0$ and $q=1$, respectively. In the case $m \geqslant 1$ and $q \geqslant 3$ odd, using this characterization and the standard techniques of [BGMY], one can recover the best of the known lower bounds of the topological entropy.

3. Unimodal maps. Statement of results. Here and in $\$ 5$ we assume that the notation and standard techniques of part II of [CE] are known to the reader, and we freely use them in these sections.

Definition 3.1. We say that a map $f \in C([a, b])$ is unimodal if:

(1) $f(c)=b$ for some $c \in(a, b)$,

(2) $f$ is strictly decreasing on $[c, b]$ and strictly increasing on $[a, c]$.

Let $P=\left\{p_{1}, p_{2}, \ldots, p_{n}\right\}$ be a periodic orbit of a unimodal map $f$ with $p_{1}<p_{2}<$ $\cdots<p_{n}$, and let $p_{s}$ be the point of $P$ such that $f\left(p_{s}\right)=p_{n}$. Then we have either $c \in\left(p_{s-1}, p_{s}\right)$ and $I_{n-1}\left(p_{n}\right)=R$, or $c=p_{s}$ and $I_{n-1}\left(p_{n}\right)=C$, or $c \in\left(p_{s}, p_{s+1}\right)$ and $I_{n-1}\left(p_{n}\right)=L$ (where $I_{n-1}\left(p_{n}\right)$ is the $n$th symbol of the itinerary of $p_{n}, \underline{I}\left(p_{n}\right)$ ). To consider these three possibilities we shall need the following.

Definition 3.2. Let $\underline{P}_{n}=I_{0} I_{1} \cdots I_{n-1}$ be the min-max of length $n$. We denote by $\underline{P}_{n}^{c}$ and $\underline{P}_{n}^{\prime}$ the sequences $I_{0} I_{1} \cdots I_{n-2} C$ and $I_{0} I_{1} \cdots I_{n-2} I_{n-1}^{\prime}$, respectively (we recall that $I_{n-1}^{\prime}=L$ (resp., $\left.R, C\right)$ if $I_{n-1}=R$ (resp., $\left.L, C\right)$ ). $\underline{P}_{n}^{c}$ and $\underline{P}_{n}^{1}$ will be called the $n$-quasi-min-max ( $n$-QMM). We note that if $n=2^{m}$ with $m \geqslant 1$, then $\underline{P}_{n}^{\prime}=$ $\underline{P}_{n / 2} \underline{P}_{n / 2}$. Let $f$ be a unimodal map. We say that a periodic orbit $\left\{p_{1}, p_{2}, \ldots, p_{n}\right\}$ of period $n \geqslant 2$, with $p_{1}<p_{2}<\cdots<p_{n}$ is a quasi-min-max periodic orbit (QMMPO) if $\underline{I}\left(p_{n}\right)$ is either $\underline{P}_{n}^{c}$ or $\underline{P}_{n}^{\infty}$ or $\left(\underline{P}_{n}^{\prime}\right)^{\infty}$ (see Figure 6). Note that if $n>1$ is a power of two and $\underline{I}\left(p_{n}\right)=\left(\underline{P}_{n}^{\prime}\right)^{\infty}$, then $\underline{I}\left(p_{n}\right)$ has period $n / 2$. Otherwise, for all the QMMPO of period $n$ we have that $\underline{I}\left(p_{n}\right)$ is either finite of length $n$ (if $\left.\underline{I}\left(p_{n}\right)=\underline{P}_{n}^{c}\right)$ or periodic of period $n$ (if $\underline{I}\left(p_{n}\right)$ is either $\underline{P}_{n}^{\infty}$ or $\left.\left(\underline{P}_{n}^{\prime}\right)^{\infty}\right)$. Let $\bar{f}$ be the restriction to $\left[p_{1}, p_{n}\right]$ of the $P$-linearization of $f$. We note that $P$ is a QMMPO if and only if $\underline{I}_{f}\left(p_{n}\right)=\underline{P}_{n}^{c}$.

REMARK 3.3. If $P$ is a WSPO of a unimodal map $f$, then $P$ is a SPO. Therefore, from Theorem 2.6 and Proposition 2.13 it follows that if $P$ is a MPO of $f$, then $P$ is simple.

Now we can state the following results for unimodal maps.

THEOREM 3.4. Let $f$ be a unimodal map. Then $P$ is a SPO of period $n=2^{m} q$ with $m \geqslant 0$ and $q \geqslant 1$ odd if and only if $P$ is a $Q M M P O$. Furthermore, the number of nonisomorphic SPO of period $n$ for the unimodal maps is 1 .

We note that Remark 3.3, Theorems 2.6, 2.7 and 3.4 and Proposition 2.7 state the "equivalence" between the notions of MPO, SPO and min-max for unimodal maps.

THEOREM 3.5. Let $f$ be a unimodal map. Then for every $n \in P(f)$ there exists at least one SPO of period $n$.

In the proof of Theorem 3.4 we give a way to explicitly compute the unique possible behavior of a SPO of period $n$ for the unimodal maps. In $\$ 5$ (see Proposition 5.8) we characterize this behavior from the point of view of the graph of f. 
4. Minimality and simple periodic orbits. Proofs of the results.

DEFINITION 4.1 (SEE [B12 AND BGMY]). Let $A=\left\{J_{0}, J_{1}, \ldots, J_{n}\right\}$ be a partition of $I$ into subintervals, i.e. a family of closed intervals such that $J_{0} \cup J_{1} \cup \cdots \cup J_{n}=I$, and if $i \neq k$ then $J_{i} \cap J_{k}$ consists of at most one point. Let $f \in C(I)$. We say that an interval $J$-covers $L$ (or equivalently $J \rightarrow L$ ) if there exists a closed subinterval $K$ of



$\underline{\mathrm{P}}_{5}=\mathrm{RLR}^{3}=\mathrm{RLRRR}$

$\underline{\mathrm{p}} \stackrel{\mathrm{C}}{-}=\operatorname{RLRRC}=\underline{\mathrm{I}}\left(\mathrm{p}_{5}\right)$

(a)

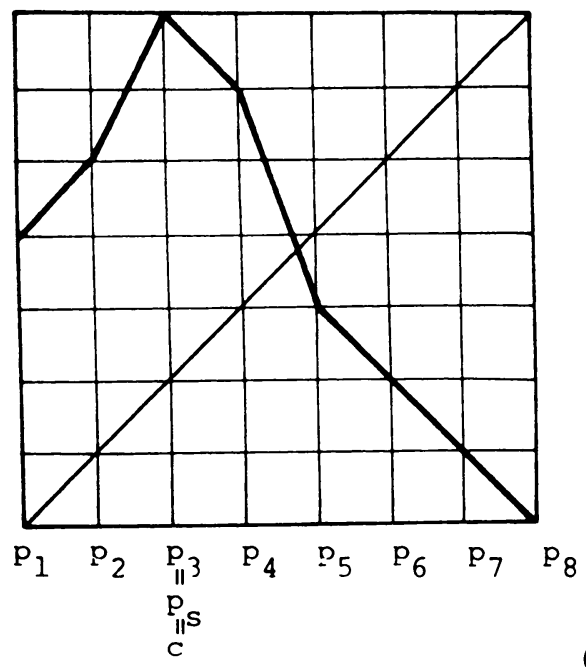

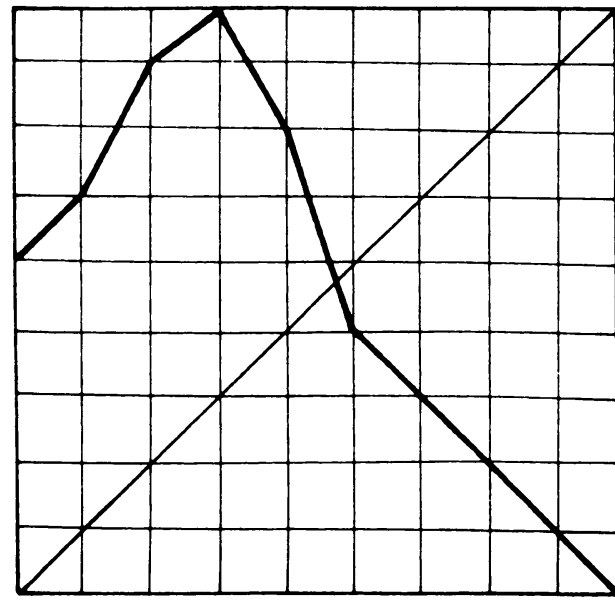

$\begin{array}{llllllllll}p_{1} & p_{2} & p_{3} & p_{4} & p_{5} & p_{6} & p_{7} & p_{8} & p_{9} & p_{10} \\ p_{s} s & & & & & & & & & \end{array}$ $\underline{\underline{P}}_{10}=\mathrm{R}^{\star} \mathrm{RLR}^{3}=$ RLRRRLRLRL $\underline{\mathrm{P}}_{10}^{\mathrm{C}}=\mathrm{RLRRRLRLRC}=\underline{\mathrm{I}}\left(\mathrm{D}_{10}\right)$

(b)

$$
\begin{aligned}
& \underline{\mathrm{P}}_{8}=\mathrm{R}^{\star}{ }^{*}{ }_{\mathrm{R}}=\mathrm{RLRRRLRL} \\
& \underline{\mathrm{P}}_{8}^{\mathrm{C}}=\mathrm{RLRRRLRC}=\underline{\mathrm{I}}\left(\mathrm{p}_{8}\right)
\end{aligned}
$$

(c)

FIgURE 6. These pictures show some QMMPO of several periods. All these orbits are of type $\underline{P}_{n}^{c}$. To obtain one of the other two types it is enough to change the position of the maximum of the map slightly to the right or to the left of $p_{s}=c$. 
$J$ such that $f(K)=L$. We say that $J f$-covers $L m$ times if there exist $m$ closed subintervals $K_{1}, K_{2}, \ldots, K_{m}$ of $J$ with pairwise disjoint interior, such that $f\left(K_{i}\right)=L$ for $i=1,2, \ldots, m$. An $A$-graph, $G$, of $f$ is an oriented generalized (i.e. possibly with several arrows joining the same vertices) graph with $n+1$ vertices $J_{0}, J_{1}, \ldots, J_{n}$ and such that if $J_{i} f$-covers $J_{k} m$ times, but not $m+1$ times, then there are $m$ (but not $m+1)$ arrows from $J_{i}$ to $J_{k}$.

LEMMA 4.2 (SEE [B12 AND BGMY]). Let $f \in C(I)$. If $J_{0} \rightarrow J_{1} \rightarrow J_{2} \rightarrow \cdots \rightarrow J_{n-1}$ $\rightarrow J_{0}$ is a loop in the A-graph of $f$, then there exists a fixed point $x$ of $f^{n}$ such that $f^{i}(x) \in J_{i}$ for $i=0,1, \ldots, n-1$.

Lemma 4.3. Let $f \in C(I)$ and let $P$ be a periodic orbit of period $n$. Suppose $f$ is $P$-linear and $A$ is the partition of $I$ given by $P$. Then the following statements hold.

(1) For every loop $J_{0} \rightarrow J_{1} \rightarrow J_{2} \rightarrow \cdots \rightarrow J_{k-1} \rightarrow J_{0}$ of the A-graph of $f$, there exists a unique fixed point $x$ of $f^{k}$, such that $f^{i}(x) \in J_{i}$ for $i=0,1, \ldots, k-1$.

(2) For every periodic point $x \notin P$ of $f$ of period $k$, there exists a unique loop $J_{0} \rightarrow J_{1} \rightarrow J_{2} \rightarrow \cdots \rightarrow J_{k-1} \rightarrow J_{0}$ of the A-graph of $f$ such that $f^{i}(x) \in J_{i}$ for $i=$ $0,1, \ldots, k-1$.

Proof. (1) Let us define the following sets: $K_{k}=J_{0}$ and $K_{i}=f^{-1}\left(K_{i+1}\right) \cap J_{i}$ for $i=k-1, k-2, \ldots, 0$. Since $f$ is $P$-linear, then each $K_{i}$ is a closed interval. Moreover, $f^{k}\left(K_{0}\right)=K_{k}$ and $\left.f^{k}\right|_{K_{0}}$ is linear. By Lemma 4.2, $f^{k}$ has a fixed point $x$ in $K_{0}$. By linearity it is unique. Clearly we have $f^{i}(x) \in J_{i}$ for $i=0,1, \ldots, k-1$.

(2) Since $x \notin P$, for every $i=0,1, \ldots, k$ we have $f^{i}(x) \in \operatorname{Int}\left(J_{r_{i}}\right)$ for some $J_{r_{1}} \in A$. Hence $f\left(\operatorname{Int}\left(J_{r_{i}}\right)\right) \cap \operatorname{Int}\left(J_{r_{i+1}}\right) \neq \varnothing$. Since $f$ is $P$-linear we have that $J_{r_{i}} f$-covers $J_{r_{i+1}}$ and $J_{r_{0}} \rightarrow J_{r_{1}} \rightarrow J_{r_{2}} \rightarrow \cdots \rightarrow J_{r_{k-1}} \rightarrow J_{r_{0}}$ is a loop of the $A$-graph of $f$. Q.E.D.

Statement (1) of the following lemma is a corollary of Lemma 5 of [LR], and (2) is Lemma 2.1 of [BGMY].

LeMMA 4.4. Suppose $P=\left\{p_{1}, p_{2}, \ldots, p_{n}\right\}$, with $p_{1}<p_{2}<\cdots<p_{n}$, is a periodic orbit of period $n$ of a map $f \in C\left(\left[p_{1}, p_{n}\right]\right)$. Let $A$ be the partition of $\left[p_{1}, p_{n}\right]$ given by the points of $P$. Then we have:

(1) If $P$ is a SPO, $f$ is $P$-linear and $n=2^{m}$ with $m \geqslant 1$, then all the loops of the $A$-graph of $f$ have length $2^{k}$ with $k<m$. Moreover, for every $k<m$ there exists a unique loop of the A-graph of $f$ of length $2^{k}$.

(2) If $P$ is a MPO of $f$ and $n \geqslant 3$ is odd, then the A-graph of $f$ has the following subgraph:

(2.1) $I_{1} \rightarrow I_{2} \rightarrow I_{3} \rightarrow \cdots \rightarrow I_{n-1} \rightarrow I_{1}$,

(2.2) $I_{n-1} f$-covers $I_{k}$ with $k=3,5,7, \ldots, n-2$.

LEMMA 4.5. Let $P=\left\{p_{1}, p_{2}, \ldots, p_{n}\right\}$, with $p_{1}<p_{2}<\cdots<p_{n}$, be a MPO of $f \in C(I)$ of period $n=2^{m} q$ with $m \geqslant 1$ and $q \geqslant 3$ odd. Then $f\left(\left\{p_{1}, p_{2}, \ldots, p_{n / 2}\right\}\right)=$ $\left\{p_{(n / 2)+1}, \ldots, p_{n}\right\}$.

Proof. Let $A$ be the partition of the interval $\left[p_{1}, p_{n}\right]$ by the subintervals $I_{k}=\left[p_{k}, p_{k+1}\right]$ for $k=1,2, \ldots, n-1$. Clearly $f\left(p_{n}\right)<p_{n}$. Hence there exists an element $I_{k_{1}}$ of $A$ whose left endpoint is the largest $p_{i}$ such that $f\left(p_{i}\right)>p_{i}$. Then 
$f\left(p_{k_{1}+1}\right) \leqslant p_{k_{1}}$ and, hence, $f\left(I_{k_{1}}\right) \supset I_{k_{1}}$. Therefore, the sequence $\left(f^{k}\left(I_{k_{1}}\right)\right)_{k=0}^{\infty}$ is such that

$$
f^{k+1}\left(I_{k_{1}}\right) \cap\left[p_{1}, p_{n}\right] \supset f^{k}\left(I_{k_{1}}\right) \cap\left[p_{1}, p_{n}\right] .
$$

Consequently, $f^{n-2}\left(I_{k_{1}}\right) \supset\left[p_{1}, p_{n}\right]$ because the cardinality of $f^{k}\left(I_{k_{1}}\right) \cap P$ is at least $k+2$, for $k=1,2, \ldots, n-2$ (we can assume $n>2$ because if $n=2$ the proof of the lemma is immediate).

Let $J_{1}=I_{k_{1}}$. For $k=2,3, \ldots$, we define $J_{k}$ as the interval $\left[a_{k}, b_{k}\right]$ where $a_{k}=$ $\min \left\{f\left(J_{k-1} \cap P\right)\right\}$ and $b_{k}=\max \left\{f\left(J_{k-1} \cap P\right)\right\}$. Clearly, $f^{k-1}\left(J_{1}\right) \supset f\left(J_{k-1}\right) \supset J_{k}$ for $k \geqslant 2$, and $J_{k}=\left[p_{1}, p_{n}\right]$ for $k \geqslant n-1$.

We claim that $I_{k_{1}}=\left[p_{n / 2}, p_{(n / 2)+1}\right]$ and

$$
f\left(\left\{p_{1}, p_{2}, \ldots, p_{n / 2}\right\}\right)=\left\{p_{(n / 2)+1}, \ldots, p_{n}\right\} .
$$

To prove this, suppose $f\left(\left\{p_{1}, p_{2}, \ldots, p_{k_{1}}\right\}\right) \neq\left\{p_{k_{1}+1}, \ldots, p_{n}\right\}$. Then either there exists $p_{i} \in\left\{p_{1}, p_{2}, \ldots, p_{k_{1}}\right\}$ such that $f\left(p_{i}\right) \in\left\{p_{1}, p_{2}, \ldots, p_{k_{1}}\right\}$ or there exists $p_{j} \in$ $\left\{p_{k_{1}+1}, \ldots, p_{n}\right\}$ such that $f\left(p_{j}\right) \in\left\{p_{k_{1}+1}, \ldots, p_{n}\right\}$. Therefore, either $f\left(\left[p_{i}, p_{k_{1}}\right]\right) \supset I_{k_{1}}$ or $f\left(\left[p_{k_{1}+1}, p_{j}\right]\right) \supset I_{k_{1}}$. Consequently, $I_{k_{1}} \subset f\left(I_{s}\right)$ for some $I_{s} \subset\left[p_{i}, p_{k_{1}}\right]$ or $I_{s} \subset$ $\left[p_{k_{1}+1}, p_{j}\right]$.

Let $r$ be the smallest positive integer such that $I_{s} \subset J_{r}$ and let $I_{k_{r}}=I_{s}$ (clearly, $2 \leqslant r \leqslant n-1)$. Then there exists a closed subinterval $K \subset J_{r-1}$ such that $f(K)=I_{k_{r}}$ and $K \subset I_{k_{r-1}}$ for some $I_{k_{r-1}} \subset J_{r_{-1}}$. Repeating, we obtain the loop $I_{k_{1}} \rightarrow I_{k_{2}} \rightarrow$ $\cdots \rightarrow I_{k_{r}} \rightarrow I_{k_{1}}$. We may assume this loop is the shortest one (except $I_{k_{1}} \supset$ ) from $I_{k_{1}}$ to itself. If $r$ is odd, then the loop $I_{k_{1}} \rightarrow I_{k_{2}} \rightarrow \cdots \rightarrow I_{k_{r}} \rightarrow I_{k_{1}}$ gives us, by Lemma 4.2 a periodic point of $f$ of period $r$. This contradicts our assumptions. If $r$ is even, then the loop $I_{k_{1}} \rightarrow I_{k_{2}} \rightarrow \cdots \rightarrow I_{k_{r}} \rightarrow I_{k_{1}} \rightarrow I_{k_{1}}$ gives us, again, a contradiction (recall that $2 \leqslant r \leqslant n-1$ and $n$ is even). This proves the claim. Hence Lemma 4.5 follows. Q.E.D.

Computation of $a_{m}$. Let $P$ be a SPO of period $2^{m}$ with $m>1$ and $H_{1}$ and $H_{2}$ the sets $\left\{p_{1}, p_{2}, \ldots, p_{2^{m-1}}\right\}$ and $\left\{p_{2^{m-1}+1}, \ldots, p_{2^{m}}\right\}$, respectively. From Definition 2.3, $H_{1}$ and $H_{2}$ are SPO of $f^{2}$ of period $2^{m-1}$. So, $f^{2}$ has $a_{m-1}$ different possible behaviors on $H_{1}$ and on $H_{2}$. Furthermore, since $f\left(H_{1}\right)=H_{2}$, given the behavior of $f^{2}$ on $H_{1}$ and on $H_{2}$, for every possibility of $f\left(p_{1}\right)$ we will have a different shape of $f$ on $P$. Therefore $a_{m}=2^{m-1} \cdot a_{m-1} \cdot a_{m-1}$ and, since $a_{1}=1=2^{2^{1}-1-1}$, we have inductively that $a_{m}=2^{2^{m}-m-1}$. Q.E.D.

Proof of Proposition 2.7. It follows easily from Lemmas 4.3 and 4.4(1). Q.E.D.

Computation of $b_{n}$. From Remark 2.11 we note that the number of different possible behaviors of $f$ on the set $\left\{Q_{i}: i=1,2, \ldots, 2^{m}\right\}$ is $a_{m}$. Moreover, we can associate to each $Q_{i}$ the sign + or - according as $f^{2^{m}}$ is a $\mathrm{SPO}^{+}$or a $\mathrm{SPO}^{-}$on $Q_{i}$. Then, if there are $k Q_{i}$ 's, with $0 \leqslant k \leqslant 2^{m}$, associated to a + sign we have $2^{m}-k$ $Q_{i}$ 's associated to a - sign. Therefore, we can distribute them in

$$
\frac{2^{m} !}{k !\left(2^{m}-k\right) !}=\left(\begin{array}{c}
2^{m} \\
k
\end{array}\right)
$$


different ways. Then, the total number of possibilities of the distribution of signs is

$$
\sum_{k=0}^{2^{m}}\left(\begin{array}{c}
2^{m} \\
k
\end{array}\right)=2^{2^{m}}
$$

Finally, the behavior of $f$ on $P$ depends on the images $f\left(p_{1}^{i}\right)$ for $i=1,2, \ldots, 2^{m}-1$. Since we have $q$ possibilities to assign every $f\left(p_{1}^{i}\right)$, we have

$$
b_{n}=a_{m} \cdot 2^{2^{m}} \cdot q^{2^{m}-1}=2^{2^{m+1}-m-1} \cdot q^{2^{m}-1} . \quad \text { Q.E.D. }
$$

Proof of Proposition 2.13. Suppose the period of $P$ is $n=2^{m} q$ with $m \geqslant 1$ and $q \geqslant 3$ odd. We prove Proposition 2.13 inductively. If $m=1$, by Lemma 4.5 and Theorem 2.6(1), Proposition 2.13 follows. Let $m>1$ and suppose Proposition 2.13 is true for $m-1$, that is, $n / 2=2^{m-1} q$. Then, by Lemma 4.5, Proposition 2.13 follows for $n$. Q.E.D.

The next proposition gives the characterization of a SPO $P$ of a map $f$, in terms of the behavior of $f$ on $P$.

Proposition 4.6. Let $P$ be a SPO of $f \in C(I)$ of period $2^{m} q$ with $m \geqslant 1$ and $q \geqslant 3$ odd. Then there is a unique $Q_{r}$ which satisfies $f \equiv R\left(Q_{r}\right), f \neq-R\left(Q_{r}\right)$ and $f\left(Q_{r}\right)=Q_{s}$. Let $t=(q+1) / 2$ and $w=2^{m}$. Then only one of the following statements holds.

$$
\begin{array}{cl}
f\left(p_{t-k}^{r}\right)=p_{t+k+1}^{s} & \text { for } k=0,1, \ldots, t-2, \\
f\left(p_{t+k}^{r}\right)=p_{t-k}^{s} & \text { for } k=1,2, \ldots, t-1, \text { and } \\
f\left(p_{1}^{r}\right)=p_{t}^{s} & (\text { see Figure } 7(\mathrm{a})) . \\
f\left(p_{t-k}^{r}\right)=p_{t+k}^{s} & \text { for } k=1,2, \ldots, t-1, \\
f\left(p_{t+k}^{r}\right)=p_{t-k-1}^{s} & \text { for } k=0,1, \ldots, t-2, \text { and } \\
f\left(p_{q}^{r}\right)=p_{t}^{s} & (\text { see Figure } 7(\mathrm{~b})) . \\
f\left(p_{k}^{r}\right)=p_{k}^{s} \quad \text { for } k=1,2, \ldots, t-1, \\
f\left(p_{k}^{r}\right)=p_{k+1}^{s} \quad \text { for } k=t, t+1, t+2, \ldots, q-1, \text { and } \\
f\left(p_{q}^{r}\right)=p_{t}^{s} \quad(\text { see Figure } 7(\mathrm{c})) . \\
f\left(p_{k}^{r}\right)=p_{k-1}^{s} \quad \text { for } k=2,3, \ldots, t, \\
f\left(p_{k}^{r}\right)=p_{k}^{s} \quad \text { for } k=t+1, t+2, t+3, \ldots, q, \text { and } \\
\left.f\left(p_{1}^{r}\right)=p_{t}^{s} \quad \text { (see Figure } 7(\mathrm{~d})\right) .
\end{array}
$$

Furthermore, the following statements hold.

(5) Let $S$ be a subset of $\{1,2, \ldots, w\}$ defined by

$$
S=\left\{i \in N: f \equiv-R\left(Q_{i}\right) \text { and } i=1,2, \ldots, w\right\} .
$$

Then $|S|$ is even if and only if $f$ satisfies (1) or (2) on $Q_{r}$; and $|S|$ is odd if and only if $f$ satisfies (3) or (4) on $Q_{r}$.

(6) Let $i \in\{1,2, \ldots, w\}$. Let $k_{i}$ be the smallest positive integer such that $f^{k_{i}}\left(Q_{r}\right)=$ $Q_{i}$; of course $k_{i} \leqslant w$. We denote by $S_{i}$ the set

$$
\left\{j \in N: f \equiv-R\left(Q_{j}\right) \text { with } Q_{j}=f^{k}\left(Q_{r}\right) \text { for } k=1,2, \ldots, k_{i}-1\right\} \text {. }
$$


(a) $Q_{i}$ is a $\mathrm{SPO}^{+}$of $f^{w}$ if and only if either $\left|S_{i}\right|$ is even and $f$ satisfies (1) or (3) on $Q_{r}$, or $\left|S_{i}\right|$ is odd and $f$ satisfies (2) or (4) on $Q_{r}$.

(b) $Q_{i}$ is a SPO- of $f^{w}$ if and only if either $\left|S_{i}\right|$ is even and $f$ satisfies (2) or (4) on $Q_{r}$, or $\left|S_{i}\right|$ is odd and $f$ satisfies (1) or (3) on $Q_{r}$.

(c) $Q_{r}$ is a SPO ${ }^{+}$of $f^{w}$ if and only if $f$ satisfies (1) or (4) on $Q_{r}$.

(d) $Q_{r}$ is a SPO- of $f^{w}$ if and only if $f$ satisfies (2) or (3) on $Q_{r}$.

Proof. Suppose $|S|$ is even. Now we shall prove that $f$ satisfies either (1) or (2). In the case $|S|$ is odd, we obtain similarly that $f$ satisfies either (3) or (4).

By Remark 2.11, we have $f^{w}\left(Q_{s}\right)=Q_{s}, f^{w-1}\left(Q_{s}\right)=Q_{r}$ and $Q_{s}$ is a SPO of $f^{w}$. Since $|S|$ is even, we have $f^{w-1}\left(p_{k}^{s}\right)=p_{k}^{r}$ for $k=1,2, \ldots, q$. Now we consider two cases.

Case A. $Q_{s}$ is a $\mathrm{SPO}^{+}$for $f^{w}$.

Case B. $Q_{s}$ is a $\mathrm{SPO}^{-}$for $f^{w}$.

We shall obtain (1) from Case A, and similarly we could obtain (2) from Case B.

Since $Q_{s}$ is a $\mathrm{SPO}^{+}$for $f^{w}$, we have

$$
\begin{array}{ll}
f^{w}\left(p_{t-k}^{s}\right)=p_{t+k+1}^{s} & \text { for } k=0,1, \ldots, t-2, \\
f^{w}\left(p_{t+k}^{s}\right)=p_{t-k}^{s} & \text { for } k=1,2, \ldots, t-1, \text { and } \\
f^{w}\left(p_{1}^{s}\right)=p_{t}^{s} . &
\end{array}
$$

Therefore,

$$
\begin{array}{ll}
f\left(p_{t-k}^{r}\right)=p_{t+k+1}^{s} & \text { for } k=0,1, \ldots, t-2, \\
f\left(p_{t+k}^{r}\right)=p_{t-k}^{s} & \text { for } k=1,2, \ldots, t-1, \text { and } \\
f\left(p_{1}^{r}\right)=p_{t}^{s} . &
\end{array}
$$

Then $f$ satisfies (1) on $Q_{r}$. Now (5) follows from the proofs of (1)-(4).

(6) We shall prove the "only if" part of (a) and (b) when $f$ satisfies (1). In a similar way, it follows the "only if" part of (a) and (b) when $f$ satisfies (2), (3) or (4). Therefore, (a) and (b) hold.

To prove this we note the following facts:

(F.1) From Definition 2.15 we have $f^{w-k_{t}}\left(p_{t}^{i}\right)=p_{t}^{r}$.

(F.2) Since $f$ satisfies (1), we obtain $f\left(p_{t}^{r}\right)=p_{t+1}^{s}$

(F.3) If $\left|S_{i}\right|$ is even, then $f^{k_{1}-1}\left(p_{t+1}^{s}\right)=p_{t+1}^{i}$. If $\left|S_{i}\right|$ is odd, then $f^{k_{t}-1}\left(p_{t+1}^{s}\right)=p_{t-1}^{i}$.

(F.4) $Q_{i}$ is a SPO of period $q$ of $f^{w}$.

Then, from (F.1)-(F.3) we have $f^{w}\left(p_{t}^{i}\right)=p_{t+1}^{i}$ if $\left|S_{i}\right|$ is even and $f^{w}\left(p_{t}^{i}\right)=p_{t-1}^{i}$ if $\left|S_{i}\right|$ is odd. From Definition 2.3 and (F.4), it follows that $Q_{i}$ is a SPO ${ }^{+}$if $\left|S_{i}\right|$ is even and a SPO- if $\left|S_{i}\right|$ is odd. This completes the proof of (a) and (b).

Since $S_{r}=S$, (c) and (d) follow from (a), (b) and (5). Q.E.D.

Computation of $c_{m}$. As in the computation of $b_{n}$ we have $a_{m}$ different possible behaviors of $f$ on the set $\left\{Q_{i}: i=1,2, \ldots, w\right\}$. Now we consider two possibilities: $|S|$ even or $|S|$ odd. We shall compute the number of different possible distributions of the $Q_{i}$ 's according as $f \equiv R\left(Q_{i}\right), f \equiv-R\left(Q_{i}\right)$ or $f$ is neither $R\left(Q_{i}\right)$ nor $-R\left(Q_{i}\right)$, for the case $|S|$ even. In the proof it will be clear that this number coincides with the corresponding one for the case $|S|$ odd. 
Suppose $|S|=k$, even. Obviously we have $w-k-1 Q_{i}$ 's such that $f \equiv R\left(Q_{i}\right)$ and only one $Q_{r}$ such that $f \equiv R\left(Q_{r}\right)$ and $f \equiv-R\left(Q_{r}\right)$. From Proposition 4.6(5) we have that $f$ has two possible behaviors on $Q_{r}$. Furthermore, we can put $Q_{r}$ on $w$ different places. On the other hand, the number of possibilities of distributions of the other $w-1 Q_{i}$ 's, according as $f \equiv R\left(Q_{i}\right)$ or $f \equiv-R\left(Q_{i}\right)$ is

$$
\frac{(w-1) !}{k !(w-k-1) !}=\left(\begin{array}{c}
w-1 \\
k
\end{array}\right) \text {. }
$$

Then the number of different possible distributions of the $Q_{i}$ 's for the case $|S|$ even is

$$
2 \cdot w \cdot \sum_{\substack{k=0 \\
k \text { even }}}^{w-2}\left(\begin{array}{c}
w-1 \\
k
\end{array}\right)=2 \cdot 2^{m} \cdot 2^{2^{m}-2}=2^{2^{m}+m-1} .
$$

Therefore, $c_{m}=a_{m} \cdot 2 \cdot 2^{2^{m}+m-1}=2^{2^{m}-1}$.

Now, to prove Theorem 2.17 we need some definitions and lemmas.

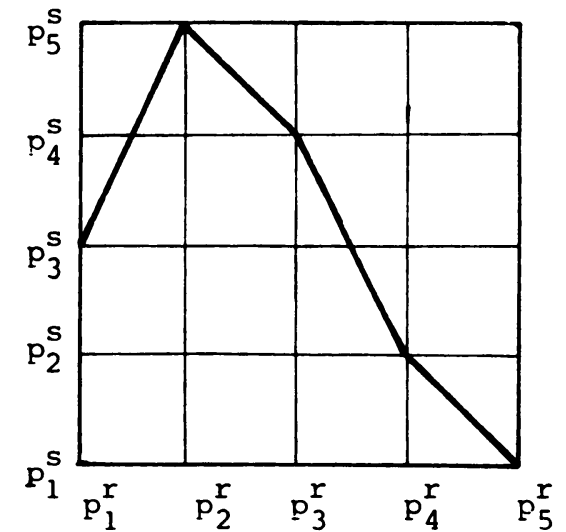

(a)

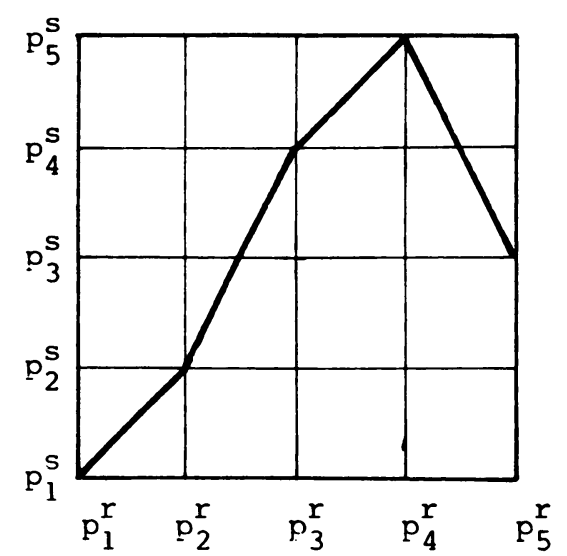

(c)



(b)

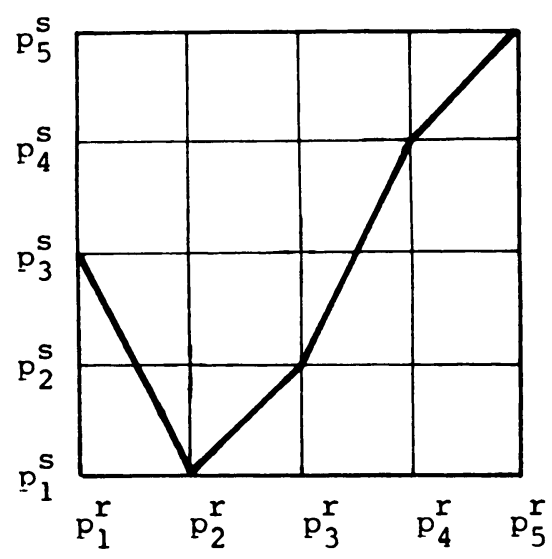

(d)

FIGURE 7. In these pictures we take $q=5$. 
Remark 4.7. Suppose $P=\left\{p_{1}, p_{2}, \ldots, p_{n}\right\}$ is a MPO of $f \in C(I)$ with period $n \geqslant 3$ odd, $p_{1}<p_{2}<\cdots<p_{n}$ and $A=\left\{I_{1}, I_{2}, \ldots, I_{n-1}\right\}$ is the partition of $\left[p_{1}, p_{n}\right]$ given by the points of $P$ (that is, $I_{i}=\left[p_{i}, p_{i+1}\right]$ for $i=1,2, \ldots, n-1$ ). Then the $A$-graph of $f$, by Lemma 4.4(2) and Theorem 2.6(1), has the following subgraph:

(a) $J_{1} \rightarrow J_{2} \rightarrow J_{3} \rightarrow \cdots \rightarrow J_{n-1} \rightarrow J_{1}$.

(b) $J_{n-1} f$-covers $J_{j}$ with $j=3,5,7, \ldots, n-2$.

(c) Furthermore, if $t=(n+1) / 2$ we have either

(c.1) $I_{t}=J_{1}, I_{t-1}=J_{2}, I_{t+1}=J_{3}, I_{t-2}=J_{4}, \ldots, I_{n-1}=J_{n-2}, I_{1}=J_{n-1}$, if $P$ is a $\mathrm{SPO}^{+}$, or

is a $\mathrm{SPO}^{-}$.

(c.2) $I_{t-1}=J_{1}, I_{t}=J_{2}, I_{t-2}=J_{3}, I_{t+1}=J_{4}, \ldots, I_{1}=J_{n-2}, I_{n-1}=J_{n-1}$, if $P$

Moreover, since $P$ is minimal the $A$-graph of $f$ must be a subgraph of a graph which satisfies (a)-(d) with

(d) $J_{i} f$-covers $J_{j}$ where $3 \leqslant i<n-1$ and $j \in\{i-1, i-3, i-5, \ldots\}$ with $j \geqslant 2$.

We note that, here, we have used facts about the length of the loops of the $A$-graph of $f$ and that if $J_{2} f$-covers $J_{2}$ then $J_{2} f$-covers $J_{1}$; which is not possible if $n>3$.

Definition 4.8. Let $f \in C(I)$ and let $A$ be a partition of $I$ into $n$ subintervals. We can associate to the $A$-graph of $f$ an $n \times n$ matrix $M=\left(m_{i j}\right)$ such that $m_{i j}=$ number of times that $I_{i} f$-covers $I_{j}$. The matrix $M$ will be called the transition matrix of the $A$-graph of $f$.

REMARK 4.9. Let $f \in C(I)$ and let $M$ be a transition matrix of an $A$-graph of $f$. Let $M^{k}=\left(m_{i j}^{k}\right)$. It is easy to show that $m_{i i}^{k} \leqslant$ number of fixed points of $f^{k}$ in the interval $I_{i}$. Therefore $\operatorname{Tr}\left(M^{k}\right) \leqslant$ number of fixed points of $f^{k}$. Furthermore, if $P$ is a periodic orbit of $f$ with period $n$ and $f$ is $P$-linear, then, from Lemma 4.3, we have $m_{i i}^{k}=$ number of fixed points of $f^{k}$ in $I_{i}$ if $n$ does not divide $k$. Therefore $\operatorname{Tr}\left(M^{k}\right)=$ number of fixed points of $f^{k}$ if $n$ does not divide $k$.

LEMMA 4.10. Let

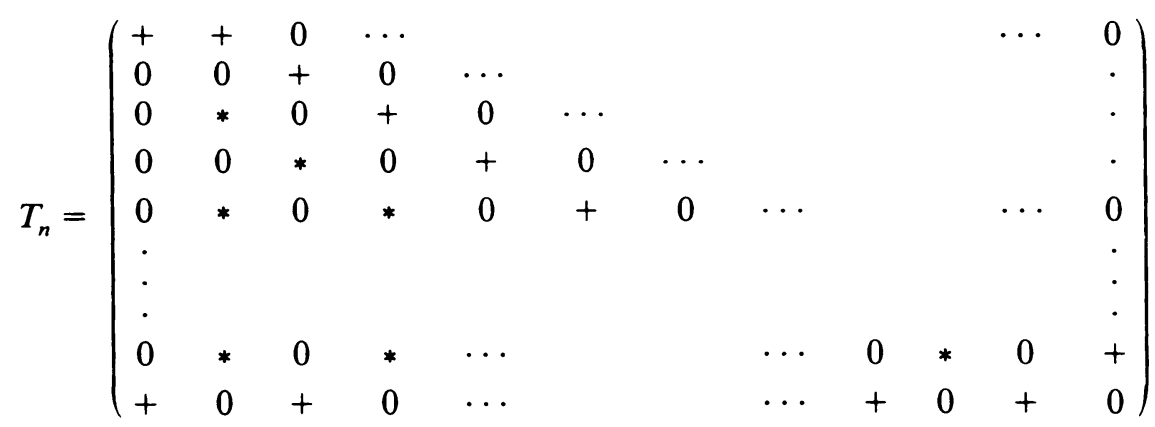

be an $n \times n$ matrix, with $n \geqslant 4$ even, where in every entry with $+($ resp. $*)$ we can put every positive (resp. nonnegative) integer. Then det $T_{n}<0$.

Proof. Obviously we have

$$
\operatorname{det} T_{n}=t_{11} \cdot \operatorname{det} T_{n}(1,1)-t_{n 1} \cdot \operatorname{det} T_{n}(n, 1)
$$


if $T_{n}=\left(t_{i j}\right)$ and $T_{n}(i, j)$ is the matrix obtained from $T_{n}$ by deleting the $i$ th row and the $j$ th column. Clearly, det $T_{n}(n, 1)$ is the product of the elements of the diagonal of $T_{n}(n, 1)$ and, thus, it is a positive integer. Now we shall prove that for every $n$ even, $\operatorname{det} T_{n}(1,1)=0$ and the lemma will follow. We note that

$$
T_{n}(1,1)=\left(\begin{array}{cccccccccc}
0 & + & 0 & \ldots & & & & & \cdots & 0 \\
* & 0 & + & 0 & \cdots & & & & . \\
0 & * & 0 & + & 0 & \cdots & & & . \\
* & 0 & * & 0 & + & 0 & \cdots & & \cdots & 0 \\
: & & & & & & & & & \vdots \\
\cdot & & & & & & & & & : \\
* & 0 & * & 0 & \cdots & & \cdots & * & 0 & + \\
0 & + & 0 & + & \cdots & & \cdots & 0 & + & 0
\end{array}\right) .
$$

To prove this let us denote by $T_{n}^{j}=\left(\bar{t}_{i j}\right)$ the following $n \times n$ matrix, with $n \geqslant 4$ even and $2 \leqslant j \leqslant n-2$ even:

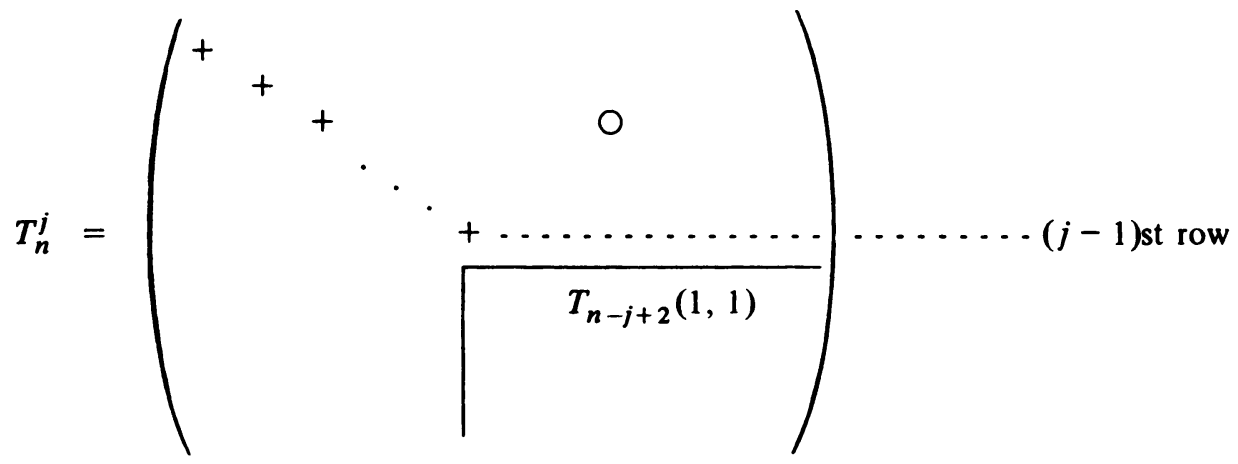

where in every entry with + we can put every positive integer. Clearly,

$$
\operatorname{det} T_{n}^{j}=\left(\prod_{i=1}^{j-1} \bar{t}_{i i}\right) \cdot \operatorname{det} T_{n-j+2}(1,1)=C\left(T_{n}^{j}\right) \cdot \operatorname{det} T_{n-j+2}(1,1),
$$

where $C\left(T_{n}^{j}\right)$ is a positive integer. Now we shall prove inductively that det $T_{n}(1,1)=0$ for $n \geqslant 4$ even.

First let $n=4$. Obviously det $T_{4}(1,1)=0$. Now let us fix $n>4$ and suppose that $\operatorname{det} T_{j}(1,1)=0$ for $j=4,6,8, \ldots, n-2$. Also we let $T_{n}(1,1)=\left(t_{i j}^{\prime}\right)$. Then we have

$$
\operatorname{det} T_{n}(1,1)=-\prod_{\substack{j=2 \\ j \text { even }}}^{n-4} t_{j 1}^{\prime} \cdot \operatorname{det} T_{n-2}^{j}-t_{n-2,1}^{\prime} \cdot \operatorname{det} T^{\prime \prime},
$$

where $T^{\prime \prime}=\left(t_{i j}^{\prime \prime}\right)$ is an $(n-2) \times(n-2)$ matrix satisfying $t_{i, n-2}^{\prime \prime}=0$ for $i=$ $1,2, \ldots, n-2$. Then $\operatorname{det} T^{\prime \prime}=0$. Moreover, by induction, we have

$$
\operatorname{det} T_{n-2}^{j}=C\left(T_{n-2}^{j}\right) \cdot \operatorname{det} T_{n-j}(1,1)=0 .
$$

Therefore det $T_{n}(1,1)=0$. Q.E.D. 
LEMMA 4.11. Let $P$ be a MPO of $f \in C(I)$ of period $n=2^{m} q$ with $m \geqslant 1$ and $q \geqslant 5$ odd, and $A$ the partition of $I$ given by the points of $P$. Moreover, suppose $f$ is $P$-linear. Then the A-graph of $f$ has the following subgraph:

$$
\begin{array}{ccccccccccc}
J_{1}^{1} & \rightarrow & J_{1}^{2} & \rightarrow & J_{1}^{3} & \rightarrow & \cdots & \rightarrow & J_{1}^{w} & \rightarrow & J_{2}^{1} \\
J_{2}^{1} & \rightarrow & J_{2}^{2} & \rightarrow & J_{2}^{3} & \rightarrow & \cdots & \rightarrow & J_{2}^{w} & \rightarrow & J_{3}^{1} \\
J_{3}^{1} & \rightarrow & \cdots & & \cdot & & & & \cdot & & \cdot \\
\cdot & & \cdot & & \cdot & & & & \cdot & & \cdot \\
\cdot & & \cdot & & \cdot & & & & \cdot & & \cdot \\
\cdot & & \cdot & & \cdot & & & & \cdots & \rightarrow & J_{q-1}^{1} \\
J_{q-1}^{1} & \rightarrow & J_{q-1}^{2} & \rightarrow & J_{q-1}^{3} & \rightarrow & \cdots & \rightarrow & J_{q-1}^{w} & \rightarrow & J_{1}^{1}
\end{array}
$$

where $w=2^{m}$ and for every $j \in\{1,2, \ldots, w\}$ there exists $i \in\{1,2, \ldots, w\}$ such that $\left\{J_{1}^{j}, J_{2}^{j}, \ldots, J_{q-1}^{j}\right\}=\left\{I_{1}^{i}, I_{2}^{i}, \ldots, I_{q-1}^{i}\right\}$ (here we use Definition 2.9).

(2) For some $i \in\{1,2, \ldots, w\}$ and $t=(q+1) / 2$ we have either

$$
I_{t}^{i}=J_{1}^{1}, I_{t-1}^{i}=J_{2}^{1}, I_{t+1}^{i}=J_{3}^{1}, I_{t-2}^{i}=J_{4}^{1}, \ldots, I_{q-1}^{i}=J_{q-2}^{1}, I_{1}^{i}=J_{q-1}^{1},
$$

or

$$
I_{t-1}^{i}=J_{1}^{1}, I_{t}^{i}=J_{2}^{1}, I_{t-2}^{i}=J_{3}^{1}, I_{t+1}^{i}=J_{4}^{1}, \ldots, I_{1}^{i}=J_{q-2}^{1}, I_{q-1}^{i}=J_{q-1}^{1} .
$$

(3) $J_{1}^{1} f$-covers $J_{q-1}^{2}$.

(4) $J_{q-1}^{1} f$-covers $J_{j}^{2}$, where $j=2,4,6, \ldots, q-3$.

Proof. By Proposition 2.13 we have that $P$ is a WSPO. Therefore, since $f$ is $P$-linear and, from Remark 2.11 , for every $i, k \in\{1,2, \ldots, w\}$ such that $f\left(Q_{i}\right)=Q_{k}$, we have $f\left(G_{i}\right)=G_{k}$.

Now we claim that for every $1 \leqslant i, k \leqslant w$ such that $f\left(Q_{i}\right)=Q_{k}$, we can label the set $\left\{I_{1}^{k}, I_{2}^{k}, \ldots, I_{q-1}^{k}\right\}=\left\{J_{1}^{k}, J_{2}^{k}, \ldots, J_{q-1}^{k}\right\}$ such that the graph of $f$ from $G_{i}$ to $G_{k}$ has a subgraph which satisfies $I_{j}^{i} f$-covers $J_{j}^{k}$ for $j=1,2, \ldots, q-1$.

First we consider the $w(q-1) \times w(q-1)$ transition matrix of the $A$-graph of $f$ restricted to $\left\{I_{j}^{i}: i=1,2, \ldots, w\right.$ and $\left.j=1,2, \ldots, q-1\right\}$ as a "box matrix". That is, $M=\left(M_{i k}\right)_{i, k=1,2, \ldots, w}$, where $M_{i k}$ is a $(q-1) \times(q-1)$ matrix which is the transition matrix of the graph of $f$ from the set $\left\{I_{j}^{i}: j=1,2, \ldots, q-1\right\}$ to $\left\{I_{j}^{k}\right.$ : $j=1,2, \ldots, q-1\}$.

We note that, to prove the claim, it is sufficient to show that for every $i, k$ satisfying $f\left(Q_{i}\right)=Q_{k}$ we have $\operatorname{det} M_{i k} \neq 0$. To prove this, since $P$ is a WSPO, $f$ is $P$-linear and from Remark 2.11 we have that for every $1 \leqslant i \leqslant w$ there exists a unique $1 \leqslant k \leqslant w$ such that $f\left(Q_{i}\right)=Q_{k}$ and $M_{i k}$ is different from the zero matrix. Therefore,

$$
\operatorname{det} M= \pm \prod_{i=1}^{w} \operatorname{det} M_{i, k(i)}
$$

where $k(i)$ satisfies $f\left(Q_{i}\right)=Q_{k(i)}$. Then, to prove the claim, it is enough to show that $\operatorname{det} M \neq 0$. 
On the other hand, we denote by $M_{i k}^{w}$ the matrix of the graph of $f^{w}$ from $\left\{I_{j}^{i}\right.$ : $j=1,2, \ldots, q-1\}$ to $\left\{I_{j}^{k}: j=1,2, \ldots, q-1\right\}$, and $M^{w}$ is the transition matrix of the $A$-graph of $f^{w}$ restricted to $\left\{I_{j}^{i}: i=1,2, \ldots, w\right.$ and $\left.j=1,2, \ldots, q-1\right\}$. Again, since $P$ is a WSPO, $f$ is $P$-linear, and from Remark 2.11 we have that $M_{i k}^{w}$ is different from the zero matrix if and only if $i=k$. Therefore

$$
\operatorname{det} M^{w}=\prod_{i=1}^{w} \operatorname{det} M_{i i}^{w} \text {. }
$$

Because det $M \neq 0$ if $\operatorname{det} M^{w} \neq 0$, to prove the claim, it is enough to show that $\operatorname{det} M_{i i}^{w} \neq 0$ for $i=1,2, \ldots, w$.

Finally, since $P$ is minimal we have that every $Q_{i}$ is a MPO of period $q$ for $f^{w}$. Then, from Remark 4.7 we obtain det $M_{i i}^{w}= \pm \operatorname{det} T_{q-1}$, where $T_{q-1}$ is defined as in Lemma 4.10. Thus, from Lemma 4.10, det $M_{i i}^{w} \neq 0$ and this proves the claim.

Now we can properly label the sets $\left\{Q_{i}: i=2,3, \ldots, w\right\}$ and $\left\{I_{j}^{i}: j=1,2, \ldots, q\right.$ $-1\}$ for $i=1,2, \ldots, w$, to obtain

$$
\begin{array}{ccccccccccc}
I_{1}^{1} & \rightarrow & J_{1}^{2} & \rightarrow & J_{1}^{3} & \rightarrow & J_{1}^{4} & \rightarrow & \cdots & \rightarrow & J_{1}{ }^{n} \\
I_{2}^{1} & \rightarrow & J_{2}^{2} & \rightarrow & J_{2}^{3} & \rightarrow & J_{2}^{4} & \rightarrow & \cdots & \rightarrow & J_{2}{ }^{{ }^{*}} \\
I_{3}^{1} & \rightarrow & J_{3}^{2} & \rightarrow & J_{3}^{3} & \rightarrow & J_{3}^{4} & \rightarrow & \cdots & \rightarrow & J_{3}{ }^{w} \\
\vdots & & \vdots & & \vdots & & \vdots & & & & \vdots \\
I_{q-1}^{1} & \rightarrow & J_{q-1}^{2} & \rightarrow & J_{q-1}^{3} & \rightarrow & J_{q-1}^{4} & \rightarrow & \cdots & \rightarrow & J_{q-1}^{w}
\end{array}
$$

Furthermore, since $P$ is minimal we have that $Q_{1}$ is a MPO of period $q$ for $f^{n}$. Then, from Lemma 4.4(2) we can label the set $\left\{I_{j}^{1}: j=1,2, \ldots, q-1\right\}=\left\{J_{j}^{1}: j=\right.$ $1,2, \ldots, q-1\}$ such that $J_{j}^{1} f^{w}$-covers $J_{j+1}^{1}$ for $j=1,2, \ldots, q-2$ and $J_{q-1}^{1} f^{w}$-covers $J_{1}^{1}$. Clearly, again labeling the sets $\left\{J_{j}^{i}: j=1,2, \ldots, q-1\right\}$ for $i=1,2, \ldots, w$, we obtain (1).

To prove (2)-(4) we note that every $Q_{i}$ is a MPO of $f^{w}$. Then, by Lemma 4.4(2) and Remark 4.7, for every $i$ there exists a unique $j$ such that $J_{j}^{i} f^{w}$-covers $J_{j}{ }^{i}$. Therefore, by using (1), there exists a $J_{j}^{i}$ such that either $J_{j}^{i} f$-covers $J_{j-1}^{i+1}$ if $j>1$ or $J_{j}^{i}$ $f$-covers $J_{q-1}^{i+1}$ if $j=1$. Again, we label the sets $\left\{Q_{i}: i=1,2, \ldots, w\right\}$ and $\left\{J_{j}^{i}\right.$ : $j=1,2, \ldots, q-1\}$ for $i=1,2, \ldots, w$ to put the above $J_{j}^{i}$ in the place $J_{1}^{1}$. Obviously we obtain (3). Moreover, since $\left(\cup_{j=1}^{q-1} J_{j}^{1}\right) \cap P$ is a MPO for $f^{w}$, from Remark 4.7(c) and the choice of $J_{1}^{1}$, we obtain (2).

Finally, from Lemma 4.4(2.2) and (1) we have that for every $k \in\{2,4,6, \ldots, q-3\}$ there exists $i(k) \in\{1,2, \ldots, w\}$ such that $J_{q-1}^{i(k)} f$-covers $J_{k}^{i(k)+1}$. Now we note that $i(k)=1$ for $k=2,4,6, \ldots, q-3$. Otherwise, since $J_{1}^{1} f$-covers $J_{q-1}^{2}$, for some $k \in\{2,4,6, \ldots, q-3\}$ we could have that $J_{1}^{1} f^{w}$-covers $J_{k+1}^{1}$, and by Remark 4.7 this is not possible. Then (4) follows. Q.E.D.

LEMMA 4.12. With the hypotheses of Lemma 4.11 the A-graph of $f$ from $\left\{J_{j}^{1}\right.$ : $j=1,2, \ldots, q-1\}$ to $\left\{J_{j}^{2}: j=1,2, \ldots, q-1\right\}$ coincides exactly with the corresponding subgraph given by Lemma 4.11. Moreover, $f$ on the set $Q_{r_{1}}=\left(\bigcup_{i=1}^{q-1} J_{i}^{1}\right) \cap P$ satisfies either (1), (2), (3), or (4) of Proposition 4.6. 
Proof. By Lemma 4.11 the $A$-graph of $f$ from $C_{1}=\left\{J_{j}^{1}: j=1,2, \ldots, q-1\right\}$ to $C_{2}=\left\{J_{j}^{2}: j=1,2, \ldots, q-1\right\}$ has a subgraph which satisfies (1), (3) and (4) of Lemma 4.11. Furthermore, by Remark 4.7, the $A$-graph of $f$ on $C_{1}$ is a subgraph of a graph which satisfies (1), (3) and (4) of Lemma 4.11 and $J_{i}^{1} f$-covers $J_{j}^{2}$ with $3 \leqslant i \leqslant q-2$ and $j \in\{i-2, i-4, i-6, \ldots\}$ with $j \geqslant 1$.

Now we suppose that Lemma $4.11(2.1)$ is satisfied and we take $C_{3}=\left\{I_{j}^{r_{1}}\right.$ : $j=1,2, \ldots, q-1\}$. Then, since $f$ is $P$-linear, the $(q-1) \times(q-1)$ transition matrix $M$ of the $A$-graph of $f$ from the set $C_{3}$ to $C_{2}$ is

$$
M=\left(\begin{array}{ccccccccccccc}
0 & 1 & 0 & 1 & \cdots & & \cdots & 0 & 1 & 0 & 1 & 0 & 1 \\
0 & + & 0 & + & \cdots & & \cdots & 0 & + & 0 & 1 & 0 & 0 \\
0 & + & 0 & + & \cdots & & \cdots & 0 & 1 & 0 & 0 & 0 & 0 \\
\vdots & \vdots & \vdots & \vdots & & & & \vdots & \vdots & \vdots & \vdots & \vdots & \vdots \\
0 & + & 0 & + & 0 & 1 & \cdots & & & \cdots & 0 & 0 & 0 \\
0 & + & 0 & 1 & 0 & 0 & \cdots & & & \cdots & 0 & 0 & 0 \\
0 & 1 & 0 & 0 & 0 & 0 & \cdots & & & \cdots & 0 & 0 & 0 \\
1 & 0 & 0 & 0 & 0 & 0 & \cdots & & & \cdots & 0 & 0 & 1 \\
+ & 0 & 1 & 0 & 0 & 0 & \cdots & & & \cdots & 0 & 0 & 0 \\
+ & 0 & + & 0 & 1 & 0 & \cdots & & & \cdots & 0 & 0 & 0 \\
\vdots & \vdots & \vdots & \vdots & \vdots & \vdots & & & & & \vdots & \vdots & \vdots \\
+ & 0 & + & 0 & \cdots & & \cdots & + & 0 & 1 & 0 & 0 & 0 \\
+ & 0 & + & 0 & \cdots & & \cdots & + & 0 & + & 0 & 1 & 0
\end{array}\right)
$$

where in every entry with + we can put zero or one (because $f$ is $P$-linear).

Let $C_{4}$ be the set $\left\{K_{j}: j=1,2, \ldots, q-1\right\}$ where $K_{1}=J_{q-2}^{2}, K_{2}=J_{q-4}^{2}, K_{3}=$ $J_{q-6}^{2}, \ldots, K_{t-2}=J_{3}^{2}, K_{t-1}=J_{1}^{2}, K_{t}=J_{q-1}^{2}, K_{t+1}=J_{2}^{2}, K_{t+2}=J_{4}^{2}, \ldots, K_{q-2}=$ $J_{q-5}^{2}, K_{q-1}=J_{q-3}^{2}$.

Then the transition matrix $M_{1}$ from $C_{3}$ to $C_{4}$ is

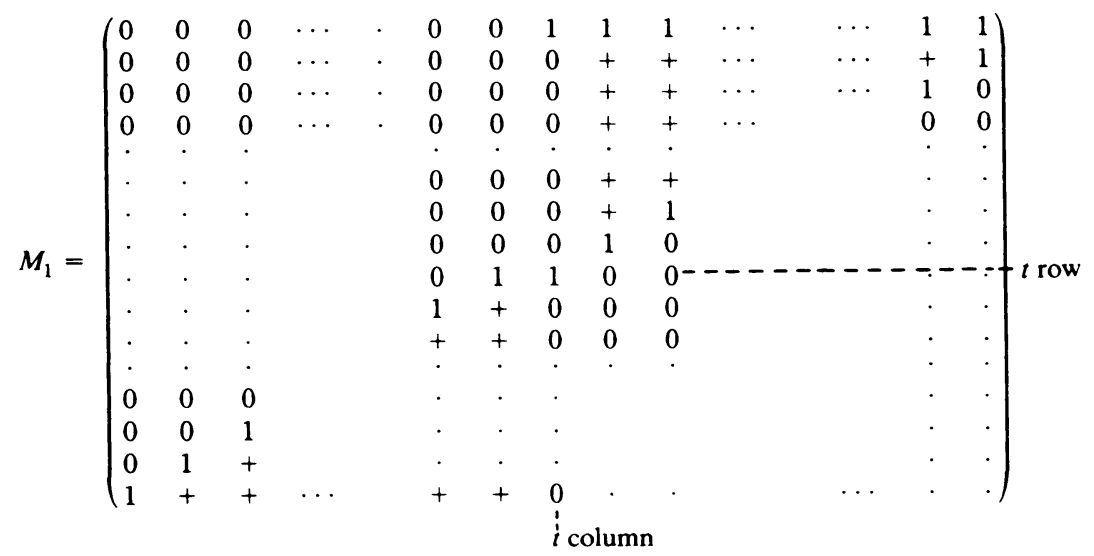

Now, since the intervals $I_{j}^{r_{1}}$ are consecutive on the real line, and $f$ is continuous and $P$-linear, we have that in every entry with + we can only put a zero. Moreover, since $f$ is continuous, the $K_{i}$ intervals are consecutive on the real line. We note that the real 
order of the $K_{i}$ intervals on the real line is either $K_{1}, K_{2}, \ldots, K_{q-1}$ or $K_{q-1}, K_{q-2}, \ldots, K_{1}$. In the first case, obviously, we have that $f$ satisfies Proposition 4.6(1) on $Q_{r_{1}}$ and in the other case $f$ satisfies Proposition 4.6(4) on $Q_{r_{1}}$.

Finally, if (2.2) of Lemma 4.11 is satisfied, we obtain similarly that $f$ satisfies either (2) or (3) of Proposition 4.6 on $Q_{r_{1}}$. Q.E.D.

LEMMA 4.13. With the hypotheses of Lemma 4.11 we have that $P$ is simple. Furthermore, the graph of $f$ on the set $\left\{I_{j}^{i}: j=1,2, \ldots, q-1\right.$ and $\left.i=1,2, \ldots, w\right\}$ coincides exactly with the graph given in Lemma 4.11.

Proof. We shall prove that for every $Q_{i}$ with $i=1,2, \ldots, w$ and $i \neq r_{1}$ either $f \equiv R\left(Q_{i}\right)$ or $f \equiv-R\left(Q_{i}\right)$, where $r_{1}$ is defined as in Lemma 4.12.

Then, from Lemmas 4.11 and 4.12 and Proposition 2.13 both statements of Lemma 4.13 follow.

From Lemma 4.12 we have that $f$ on $Q_{r_{1}}$ satisfies either (1), (2), (3), or (4) of Proposition 4.6. We shall construct the proof when $f$ satisfies Proposition 4.6(1) on $Q_{r_{1}}$. For the other cases the proof is analogous. Therefore, from the above hypotheses we see that we can write the graph given in Lemma 4.11 in the following way (where $t=(q+1) / 2$ and $Q_{r_{2}}=\left(\bigcup_{j=1}^{q-1} J_{j}^{2}\right) \cap P$ ):



Now we denote by $s, 1 \leqslant s \leqslant w$, the largest integer such that $f \neq R\left(Q_{r_{s}}\right)$ and $f \neq-R\left(Q_{r_{s}}\right)$, where $Q_{r_{t}}=\left(\bigcup_{i=1}^{q-1} J_{j}^{i}\right) \cap P$ for $i=1,2, \ldots, w$. Obviously, if $s<w$ then we have that for every integer $i$ such that $s+1 \leqslant i \leqslant w$ either $f \equiv R\left(Q_{r_{1}}\right)$ or $f \equiv-R\left(Q_{r_{1}}\right)$.

Clearly, if $s=1$ the lemma follows. Then we suppose $s \geqslant 2$. Now we shall prove that either $f \equiv R\left(Q_{r_{s}}\right)$ or $f \equiv-R\left(Q_{r_{s}}\right)$, and this will be a contradiction.

Let $C=\left\{i \in N: s+1 \leqslant i \leqslant w\right.$ and $\left.f \equiv-R\left(Q_{r_{i}}\right)\right\}$. We prove the above statement for the case $|C|$ even. The proof when $|C|$ is odd is analogous. Since $|C|$ is even, we can write the above graph as follows:

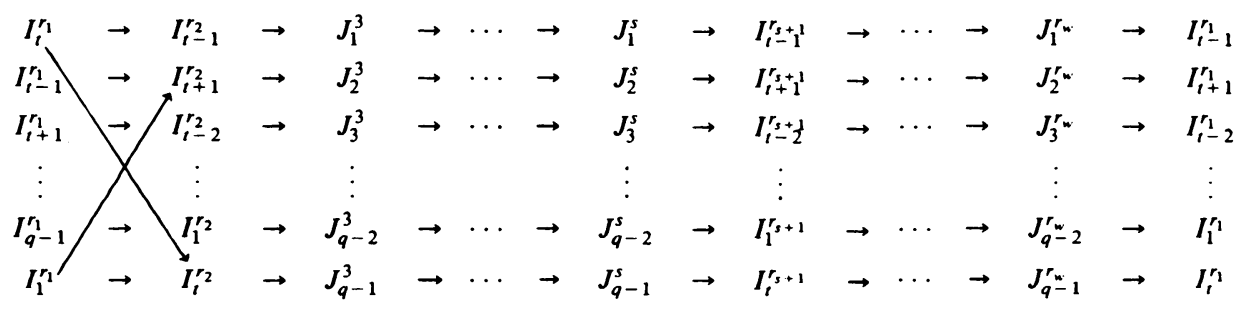


Now we have that the graph of $f$ from $\left\{J_{j}^{s}: j=1,2, \ldots, q-1\right\}$ to $\left\{I_{j}^{r_{s+1}}: j=\right.$ $1,2, \ldots, q-1\}$ is a subgraph of the following graph:

(a) $J_{j}^{s}$ f-covers $J_{j}^{s+1}$ for $j=1,2, \ldots, q-1$.

(b) $J_{i}^{s} f$-covers $J_{j}^{s+1}$ with $3 \leqslant i \leqslant q-2$ and $j \in\{i-2, i-4, i-6, \ldots\}, j \geqslant 1$.

To prove this, we note that $J_{q-1}^{s} f^{w}$-covers $J_{q-1}^{s}$. Then, from Remark 4.7 and the minimality of $P$, we have (a), (b) and

(c) $J_{q-1}^{s} f$-covers $I_{1}^{r_{s+1}}$,

(d) $J_{2}^{s} f$-covers $I_{t}^{r_{s+1}}$.

But, from (c) and (d) we obtain $I_{1}^{r_{1}} f^{w}$-covers $I_{1}^{r_{1}}$ and $I_{t-1}^{r_{1}} f^{w}$-covers $I_{t}^{r_{1}}$, respectively. Again from Remark 4.7, this is not possible.

Therefore, since $f$ is $P$-linear the $(q-1) \times(q-1)$ transition matrix $M$ of the graph of $f$ from $\left\{J_{j}^{s}: j=1,2, \ldots, q-1\right\}$ to $\left\{I_{j}^{r_{s+1}}: j=1,2, \ldots, q-1\right\}$ is

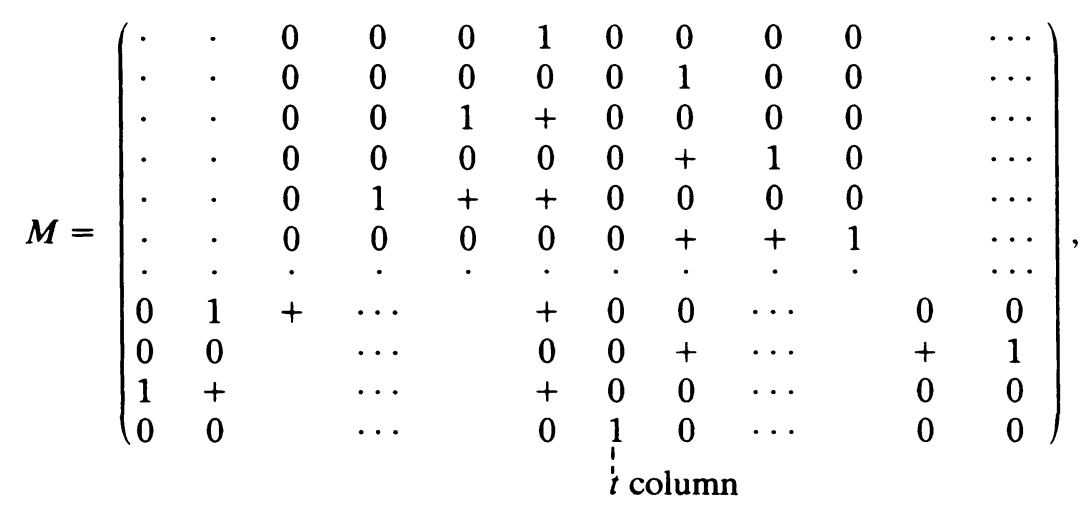

where in every entry with + we can put zero or one.

We take $L_{1}=J_{q-2}^{s}, \quad L_{2}=J_{q-4}^{s}, \ldots, L_{t-1}=J_{1}^{s}, \quad L_{t}=J_{q-1}^{s}, \quad L_{t+1}=J_{2}^{s}, \quad L_{t+2}=$ $J_{4}^{s}, \ldots, L_{q-1}=J_{q-3}^{s}$ and we have that the transition matrix of the graph of $f$ from $\left\{L_{j}: j=1,2, \ldots, q-1\right\}$ to $\left\{I_{j}^{r_{s+1}}: j=1,2, \ldots, q-1\right\}$ is given by

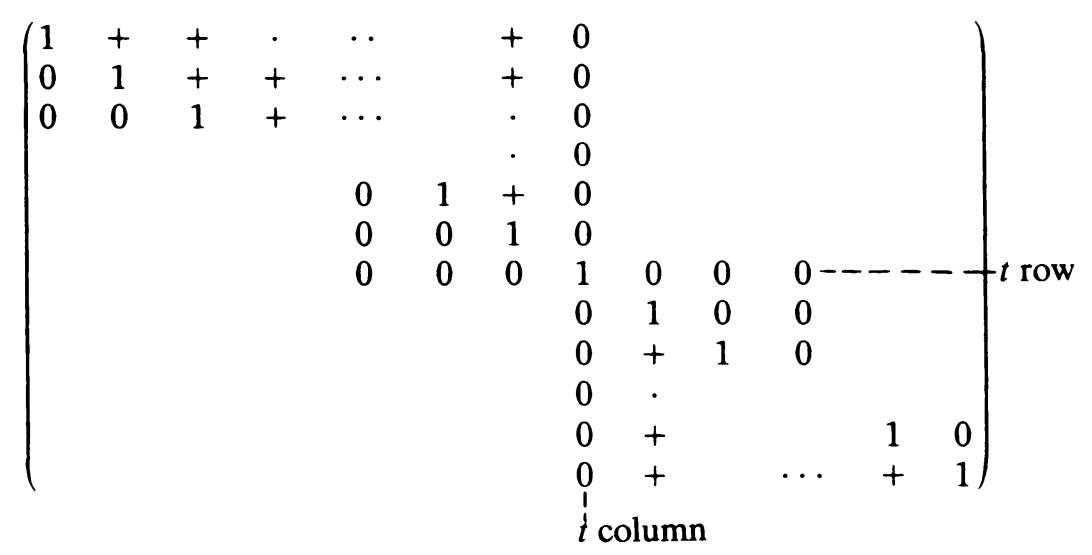

Since $f$ is continuous, $P$-linear and the intervals $I_{j}^{r_{s+1}}$ are consecutive on the real line, as in Lemma 4.12 it follows that the intervals $L_{j}$ are consecutive and its order on the real line is either $L_{1}, L_{2}, \ldots, L_{q-1}$ or $L_{q-1}, L_{q-2}, \ldots, L_{1}$, and in every entry with + we must put a zero. Therefore, $f \equiv R\left(Q_{r_{s}}\right)$ or $f \equiv-R\left(Q_{r_{s}}\right)$ according as the 
order of the $L_{j}$ intervals on the real line is $L_{1}, L_{2}, \ldots, L_{q-1}$ or the reverse one. Q.E.D.

LEMMA 4.14. Let $P$ be a WSPO of $f \in C(I)$ of period $n=2^{m} q$ with $m \geqslant 1$ and $q \geqslant 3$ odd, and let $A$ be the partition of $I$ given by the points of $P$. Moreover, suppose $f$ is $P$-linear and let $B=\left\{J \in A: J \not \subset G_{i}\right.$ for $\left.i=1,2, \ldots, w\right\}$. Then all the loops of the $A$-graph of $f$ on $B$ have length $2^{k}$ with $k<m$. Furthermore, for every $J \in B$ there does not exist $I \in A$ and $I \notin B$ such that $I$-covers $J$.

Proof. Since $f$ is $P$-linear, from Remark 2.11 we have $f\left(G_{i}\right)=G_{j}$ for every $i=1,2, \ldots, w$ and for some $j \in\{1,2, \ldots, w\}$. Hence the second statement of this lemma follows.

To prove the first statement, from Remark 2.11 we can take a map $g$ such that:

(a) $g$ has a SPO $Q=\left\{q_{1}, q_{2}, \ldots, q_{w}\right\}$ of period $w=2^{m}$ where $q_{1}<q_{2}<\cdots<q_{w}$.

(b) $g$ is $Q$-linear and $g \in C\left(\left[q_{1}, q_{w}\right]\right)$.

(c) $g\left(q_{i}\right)=q_{j}$ if and only if $f\left(Q_{i}\right)=Q_{j}$ with $i, j \in\{1,2, \ldots, w\}$.

Let $C$ be the partition of $\left[q_{1}, q_{w}\right]$ by the points of $Q$. Obviously the $A$-graph of $f$ on $B$ coincides exactly with the $C$-graph of $g$. Then Lemma 4.14 follows from Lemma 4.4(1). Q.E.D.

Proposition 4.15. Let $P$ be a SPO of a map $f \in C(I)$ of period $n=2^{m} q$ with $m \geqslant 1$ and $q \geqslant 3$ odd. Suppose $A$ is the partition of $I$ given by the points of $P$. Then the A-graph of $f$ has a subgraph which satisfies:

(a) $I_{1} \rightarrow I_{2} \rightarrow \cdots \rightarrow I_{(q-1) w} \rightarrow I_{1}$.

(b) For every $i \in\{1.2, \ldots, w\}$ there exists some $s \in\{1,2, \ldots, w\}$ such that (here we use Definition 2.9)

$$
\left\{I_{i}, I_{w+i}, I_{2 w+i}, \ldots, I_{(q-2) w+i}\right\}=\left\{I_{1}^{s}, I_{2}^{s}, \ldots, I_{q-1}^{s}\right\} .
$$

(c) $I_{w} \rightarrow I_{1}$.

(d) $I_{(q-1) w} \rightarrow I_{j w+1}$ where $j=2,4,6, \ldots, q-3$.

Let us define the intervals $J_{1}, J_{2}, \ldots, J_{w-1}$ as follows: $B=\left\{J_{1}, J_{2}, \ldots, J_{w-1}\right\}=$ $\left\{J \in A \not \subset G_{i}\right.$ for $i=1,2, \ldots, w$ and $\left.J \subset[\min P, \max P]\right\}$.

(e) All the loops of the A-graph of $f$ restricted to $B$ have length $2^{k}$ with $k<m$.

(f) If $f$ is $P$-linear, every interval $I_{i}$ with $i=1,2, \ldots,(q-1) w$ does not $f$-cover any interval $J_{j}$ with $j=1,2, \ldots, w-1$.

Proof. Let $\bar{f}$ be the $P$-linearization of $f$. Clearly, if we prove Proposition 4.15 for $\bar{f}$ it will follow for $f$. Hence, we suppose that $f$ is $P$-linear. Since $P$ is a SPO, we have that $f$ satisfies either (1), (2), (3), or (4) of Proposition 4.6 on $Q_{r}$ (here we use the notation of Proposition 4.6). We prove Proposition 4.15 when $f$ satisfies Proposition 4.6(1) on $Q_{r}$ (see Figure 8 for an example with $n=4 \cdot 5$ ). Proofs for the other cases are similar.

Now, we have that $I_{t}^{r} f$-covers $I_{t-1}^{s}, I_{t}^{s}$, and there does not exist $j \in\{1,2, \ldots, q-1\}$ with $j \neq t=(q+1) / 2$ such that $I_{j}^{r} f$-covers $I_{t-1}^{s}$. Furthermore, for every $i \in$ $\{2,3, \ldots, q-1\}-\{t\}, I_{i}^{r} f$-covers a unique interval. On the other hand, from Remark 2.11 and since $f$ is $P$-linear, we have $f\left(G_{i}\right)=G_{j}$ with $j \neq i$ and $f^{w}\left(G_{i}\right)=G_{i}$ 
for every $i=1,2, \ldots, w$. Moreover, from Definition $2.15, I_{j}^{i} f$-covers a unique interval for $i=1,2, \ldots, w, i \neq r$ and $j=1,2, \ldots, q-1$.

Therefore, from Proposition 4.6(c), we have that the $A$-graph of $f^{w}$ on the set $\left\{I_{j}^{r}\right.$ : $j=1,2, \ldots, q-1\}$ is the following:

$$
\widehat{I_{t}^{P}} \rightarrow I_{t-1}^{r} \rightarrow I_{t+1}^{r} \rightarrow I_{t-2}^{r} \rightarrow I_{t+2}^{r} \rightarrow \cdots \rightarrow I_{q-1}^{r} \rightarrow I_{1}^{r} \rightarrow I_{t}^{r}
$$

$$
I_{1}^{r} f \text {-covers } I_{j}^{r} \text { with } j=t+1, t+2, \ldots, q-1 \text {. }
$$

Then we take $I_{w}=I_{t}^{r}, I_{w+1}=I_{t-1}^{s}$, and $I_{w+k}$, with $k=2,3, \ldots, w$, the unique interval $K$ such that $I_{w+k-1} f$-covers $K$. Obviously $I_{2 w}=I_{t-1}^{r}$. Repeating this process we have $I_{2 w+1}=I_{t+1}^{s}, I_{3 w}=I_{t+1}^{r}, I_{3 w+1}=I_{t-2}^{s}, \ldots, I_{(q-1) w}=I_{1}^{r}$. Since $I_{1}^{r} f$-covers $I_{j}^{s}$

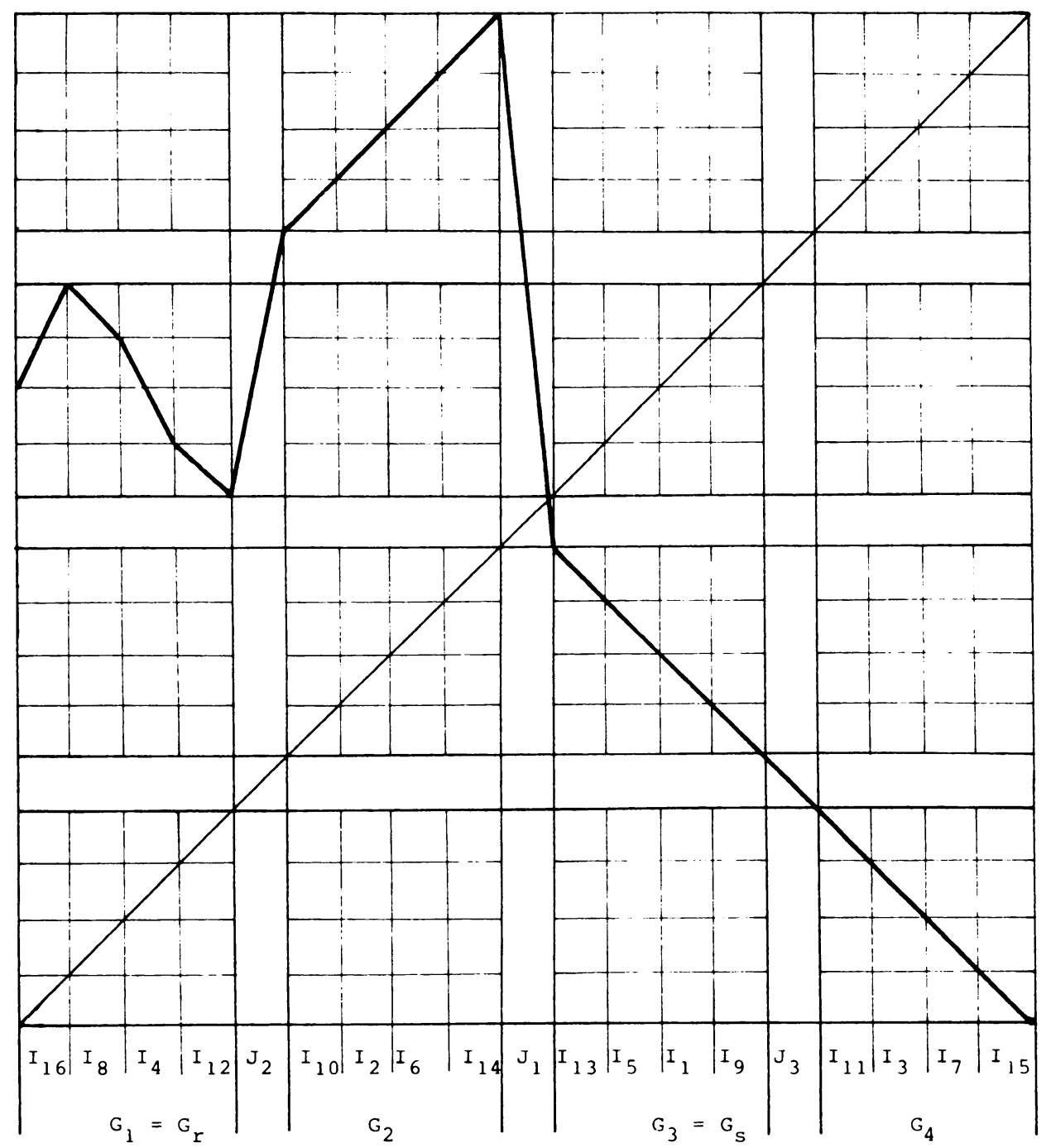


with $j \in\{t, t+1, t+2, \ldots, q-1\}$ and $\left\{I_{j}^{s}: j=t+1, t+2, \ldots, q-1\right\}=\left\{I_{w k+1}\right.$ : $k=2,4,6, \ldots, q-3\}$, we take $I_{1}=I_{t}^{s}$ and $I_{k}$, with $k=2,3, \ldots, w$, the unique interval $K$ such that $I_{k-1} f$-covers $K$. Therefore $I_{w} f$-covers $I_{1}$ and $I_{(q-1) w} f$-covers $I_{k w+1}$ with $k=2,4,6, \ldots, q-3$. Hence, we have proved (a), (c) and (d) of Proposition 4.15. Now (b) follows by construction. Finally, (e) and (f) follow from Definition 2.15 and Lemma 4.14. Q.E.D.

Proof of Theorem 2.17. Let $A$ be the partion of $I$ given by the points of $P$ and $\bar{f}$ the $P$-linearization of $f$. We remark that the $A$-graph of $\bar{f}$ is a subgraph of the $A$-graph of $f$.

(1) By Lemma 4.3, we have $P(\bar{f}) \subset P(f)$. Furthermore, from Theorem 2.1, we have that $P$ is a MPO of $\vec{f}$. We note, from Definition 2.5 , that $\bar{f}$ behaves as $f$ on $P$. Then, by Lemma 4.13, (1) follows.

(2) The $A$-graph of $f$ given by Proposition 4.15 has "generator loops" of the following lengths:

$$
\begin{array}{ll}
2^{m+1} \cdot k \text { with } k=(q-1) / 2 & \text { from (a), } \\
w & \text { from (c), } \\
2 w, 4 w, 6 w, \ldots,(q-3) w & \text { from (d), } \\
2^{k} \text { with } k \in C \text { and } C \subset\{0,1,2, \ldots, m-1\} & \text { from (e). }
\end{array}
$$

Since all these lengths are greater than $n$ in Sarkovskii's ordering, (2) follows from Lemma 4.3.

(3) By Proposition 2.13 we have that $P$ is a WSPO of $f$. Suppose $P$ is not a SPO. Then we take $\bar{f}$, the $P$-linearization of $f$, and we write the $A$-graph of $\bar{f}$ in a similar way to the proof of Lemma 4.11. The $A$-graph of $\bar{f}$ has as a subgraph:

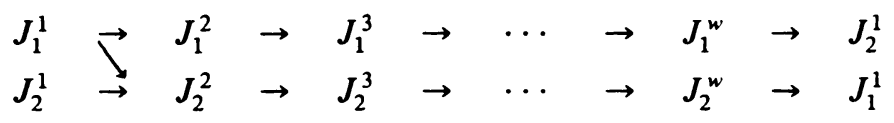

Since $\left|Q_{i}\right|=3$ for $i=1,2, \ldots, w$, we note that for every $i \in\{1,2, \ldots, w\}$ we have either $f \equiv R\left(Q_{i}\right)$ or $f \equiv-R\left(Q_{i}\right)$ or $f$ satisfies either (1), (2), (3), or (4) of Proposition 4.6 on $Q_{i}$. We set $Q_{r_{i}}=\left(J_{1}^{i} \cup J_{2}^{i}\right) \cap P$, for $i=1,2, \ldots, w$. Then it follows that $f \neq R\left(Q_{r_{1}}\right)$ and $f \neq-R\left(Q_{r_{1}}\right)$. Hence, by Definition 2.15 and since $P$ is not a SPO, we have that there is (at least) one $s \in\{2,3, \ldots, w\}$ such that $f \neq R\left(Q_{r_{s}}\right)$ and $f \neq$ $-R\left(Q_{r_{s}}\right)$. Therefore, we have $J_{1}^{s} f$-covers $J_{2}^{s+1}$ or $J_{2}^{s} f$-covers $J_{1}^{s+1}$.

Now we suppose $J_{1}^{s} f$-covers $J_{2}^{s+1}$ (for the other case the proof of (3) follows similarly). Then we have two different loops of length $2^{m} \cdot 3$, that is:

and

$$
\begin{array}{rlllllllllllllll}
J_{1}^{1} \rightarrow J_{1}^{2} & \rightarrow & J_{1}^{3} & \rightarrow & \cdots & \rightarrow & J_{1}^{s} & \rightarrow & J_{2}^{s+1} & \rightarrow & \cdots & \rightarrow & J_{2}^{\omega} & \rightarrow & J_{1}^{1} \\
& J_{1}^{2} & \rightarrow & J_{1}^{3} & \rightarrow & \cdots & \rightarrow & J_{1}^{s} & \rightarrow & J_{1}^{s+1} & \rightarrow & \cdots & \rightarrow & J_{1}^{\omega} & \rightarrow & J_{2}^{1} \\
& J_{2}^{2} & \rightarrow & J_{2}^{3} & \rightarrow & \cdots & \rightarrow & J_{2}^{s} & \rightarrow & J_{2}^{s+1} & \rightarrow & \cdots & \rightarrow & J_{2}^{\omega} & \rightarrow & J_{1}^{1}
\end{array}
$$

$$
\begin{aligned}
& J_{1}^{1} \rightarrow J_{2}^{2} \rightarrow J_{2}^{3} \rightarrow \cdots \quad \rightarrow \quad J_{2}^{s} \rightarrow J_{2}^{s+1} \rightarrow \cdots \quad \cdots \quad J_{2}^{w} \rightarrow J_{1}^{1} \\
& J_{1}^{2} \rightarrow J_{1}^{3} \rightarrow \cdots \rightarrow J_{1}^{s} \rightarrow J_{1}^{s+1} \rightarrow \cdots \rightarrow J_{1}^{w} \rightarrow J_{2}^{1} \\
& J_{2}^{2} \rightarrow J_{2}^{3} \rightarrow \cdots \rightarrow J_{2}^{s} \rightarrow J_{2}^{s+1} \rightarrow \cdots \quad \rightarrow \quad J_{2}^{w} \rightarrow J_{1}^{1}
\end{aligned}
$$


But, clearly, these loops are also loops in the $A$-graphs of $f$. Hence, by Lemma 4.2, $f$ has two periodic points of period $2^{m} \cdot 3$ whose orbits are different, and this a contradiction.

(4) As above, the $A$-graph of $f$ has the following subgraph:

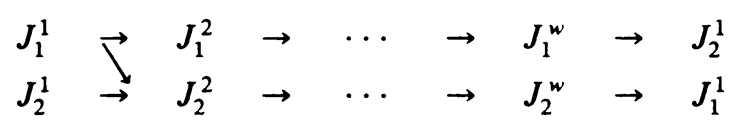

We note that every new arrow added to this graph will give us a new loop of the $A$-graph of $f$ of length $2^{m} \cdot 3$. From Lemma 4.14, all the loops of the $A$-graph of $f$ have length greater than or equal to $2^{m} \cdot 3$, in Sarkovskii's ordering. Finally, from Lemma 4.3 we have that every periodic orbit of $f$ of period $2^{m} \cdot 3$ is minimal. Then (4) follows. Q.E.D.

EXAMPLE 4.16. Here we show that the map $f$ given in Figure 4 has a WSPO $P=\left\{p_{1}, p_{2}, \ldots, p_{10}\right\}$ of period 10 such that $P$ is not a MPO.

The existence of this map tells us that the definition of WSPO is too general to describe the behavior of a MPO of period $2^{m} q$ with $m \geqslant 1$ and $q \geqslant 3$ odd. Because of this, it is necessary to use the more restrictive definition of SPO.

To show that $P$ is not a MPO, let $A=\left\{I_{1}, I_{2}, \ldots, I_{9}\right\}$ be the partition of $\left[p_{1}, p_{10}\right]$ given by the points of $P$. Then the transition matrix $M$ of the $A$-graph of $f$ is

$$
\left(\begin{array}{lllllllll}
0 & 0 & 0 & 0 & 0 & 1 & 0 & 0 & 0 \\
0 & 0 & 0 & 0 & 0 & 0 & 1 & 1 & 1 \\
0 & 0 & 0 & 0 & 0 & 0 & 0 & 1 & 1 \\
0 & 0 & 0 & 0 & 0 & 0 & 0 & 1 & 0 \\
0 & 0 & 1 & 1 & 1 & 1 & 1 & 1 & 0 \\
0 & 0 & 1 & 1 & 0 & 0 & 0 & 0 & 0 \\
0 & 1 & 1 & 1 & 0 & 0 & 0 & 0 & 0 \\
1 & 0 & 0 & 0 & 0 & 0 & 0 & 0 & 0 \\
1 & 1 & 1 & 0 & 0 & 0 & 0 & 0 & 0
\end{array}\right) .
$$

Therefore, $\operatorname{Tr}\left(M^{2}\right)=7, \operatorname{Tr}\left(M^{3}\right)=\operatorname{Tr}(M)=1$ and $\operatorname{Tr}\left(M^{6}\right)=79$. That is, from Remark 4.9, $f$ has 1 fixed point, 3 periodic orbits of period 2, and 12 periodic orbits of period 6. Thus, $P$ is not a MPO of $f$.

EXAMPLE 4.17. Figure 5 gives a map $f$ which has a minimal periodic orbit $P=\left\{p_{1}, p_{2}, \ldots, p_{6}\right\}$ of period 6 which is not simple.

This shows that Theorem 2.17(1) is only true for $q>3$. Moreover, as we prove in Theorem 2.17(3) this MPO will not be unique. To show this, let $A=\left\{I_{1}, I_{2}, \ldots, I_{5}\right\}$ be the partition of $\left[p_{1}, p_{6}\right]$, given by the points of $P$. Obviously the transition matrix $M$ of the $A$-graph of $f$ is

$$
\left(\begin{array}{lllll}
0 & 0 & 0 & 1 & 1 \\
0 & 0 & 0 & 0 & 1 \\
0 & 1 & 1 & 1 & 0 \\
1 & 0 & 0 & 0 & 0 \\
1 & 1 & 0 & 0 & 0
\end{array}\right)
$$

Now it is possible to show, inductively, that $\operatorname{Tr}\left(M^{n}\right)=1$ for every $n>3$ odd. Hence, by Remark 4.9, $f$ has no periodic points of odd period. Since $\operatorname{Tr}\left(M^{6}\right)$ $\operatorname{Tr}\left(M^{2}\right)=37-7=30, f$ has 5 MPO of period 6. 


\section{Unimodal maps. Proofs of results.}

Definition 5.1 (SEE [GM AND G]). Suppose $f$ is a unimodal map, $P=$ $\left\{p_{1}, p_{2}, \ldots, p_{n}\right\}$ is a periodic orbit of period $n \in N, p_{1}<p_{2}<\cdots<p_{n}$ and the maximum of $f, c \in P$. Then we set $q_{1}=c, q_{2}=f\left(q_{1}\right)=p_{n}, q_{3}=f\left(q_{2}\right)=p_{1}$, and $q_{i}=f\left(q_{i-1}\right)$ for $i=1,2, \ldots, n$. Obviously, if $n \geqslant 3$ we have $q_{3} \leqslant q_{i}$ for all $i$. If $q_{i_{1}}<q_{i_{2}}<\cdots<q_{i_{n}}$, then $R_{n}=\left(i_{1}, i_{2}, \ldots, i_{n}\right)$ is a permutation of the set $\{1,2, \ldots, n\} . R_{n}$ is called the rotation sequence of $P$ related with $f$. It is clear that $R_{n}=(3, \ldots, 1, \ldots, 2)$ for each $n \geqslant 3$.

LEMMA 5.2 (LEMMA 11 OF [L1]). For each $n \geqslant 2$, there is a bijection between the set of rotation sequences $R_{n}$ and the set of maximal sequences $\underline{I}$ with $|\underline{I}|=n$.

REMARK 5.3 (SEE [L1]). There is a way to compute the maximal sequence associated to $R_{n}$ and conversely. If we denote by $\underline{I}\left(R_{n}\right)$ the maximal sequence associated with the rotation sequence $R_{n}$, then $\underline{I}\left(R_{n}\right)=I_{0} I_{1} I_{2} \cdots I_{n-1}$ where

$$
I_{i}= \begin{cases}R & \text { if } 1 \text { appears before } i+2 \text { in } R_{n}, \\ L & \text { if } i+2 \text { appears before } 1 \text { in } R_{n}, \\ C & \text { otherwise }\end{cases}
$$

We denote by $R_{n}(\underline{I})$ the rotation sequence associated with the maximal sequence $\underline{I}$ with $|\underline{I}|=n$. To compute $R_{n}(\underline{I})$ we order the set of itineraries $\left\{S^{k}(\underline{I}): k=\right.$ $0,1, \ldots, n-1\}$ and obtain $S^{j_{1}}(\underline{I})<S^{j_{2}}(\underline{I})<\cdots<S^{j_{n}}(\underline{I})=\underline{I}$, where $\left(j_{1}, j_{2}, \ldots, j_{n}\right)$ is a permutation of $\{0,1, \ldots, n-1\}$. Then $R_{n}(\underline{I})=\left(i_{1}, \ldots, i_{n}\right)$ where

$$
i_{k}= \begin{cases}j_{k+2} & \text { if } j_{k+2} \neq n+1 \\ 1 & \text { otherwise. }\end{cases}
$$

REMARK 5.4 (See the definition of $R^{* n}$ and the Remark of [CE, p. 79].) $R^{* m}$ is even if and only if $m$ is even. On the other hand, $\underline{P}_{n}$ (the min-max of order $n$ ) is even for all $n$ of the form $2^{m} q, q \geqslant 3$ odd, and odd for all $n=2^{m}, m>0$.

LEMMA 5.5. Let $t, s \in N$ and suppose $t \rightarrow s$. Then the following statements hold.

(1) If $t=2^{m}$ with $m \geqslant 1$, then $\underline{P}_{s}^{\infty} \leqslant\left(\underline{P}_{t}^{\prime}\right)^{\infty}<\underline{P}_{t}^{c}<\underline{P}_{t}^{\infty}$.

(2) If $t \neq 2^{m}$ for all positive integers $m$ and $s=2^{k}$ with $k \geqslant 1$, then $\underline{P}_{s}^{c}<\underline{P}_{t}^{\infty}$.

(3) If $t \neq 2^{m}$ and $s \neq 2^{m}$ for all positive integers $m$, then $\underline{P}_{s}^{c}<\underline{P}_{t}^{\infty}$ and $\left(\underline{P}_{s}^{\prime}\right)^{\infty}<\underline{P}_{t}^{\infty}$.

Proof. (1) From Theorems II.2.9 and II.2.12 of [CE] and Definition 3.2 we have

$$
\begin{aligned}
\underline{P}_{t}^{\infty} & =\left(R^{* m} * R\right)^{\infty}>\underline{P}_{t}^{c}=R^{*(m-1)} * R C>\left(\underline{P}_{t}^{\prime}\right)^{\infty} \\
& =\underline{P}_{t / 2}^{\infty}=\left(R^{*(m-1)} * R\right)^{\infty} \geqslant \underline{P}_{s}^{\infty}=\left(R^{* k} * R\right)^{\infty},
\end{aligned}
$$

where $s=2^{k}$.

(2) From (1) and Theorem II.2.8 of [CE] it follows that $\underline{P}_{s}^{c}<\underline{P}_{s}^{\infty}<\underline{P}_{t}^{\infty}$.

(3) Let $s=2^{r} q$ with $q \geqslant 3$ odd and $r \geqslant 0$. Now we shall consider two cases.

Case A. $q>3$. We define $a(s)=2^{r}(q-2)$. Then $t \rightarrow a(s) \rightarrow s$ or $t=a(s) \rightarrow s$. First we assume that $r>0$ is even. By Theorem II.2.9 of [CE], Remark 5.4 and 
Definition 3.2 we have

$$
\begin{aligned}
& \underline{P}_{a(s)}=R^{* r} R R^{* r} L\left(R^{* r} R\right)^{q-4}, \\
& \underline{P}_{s}^{c}=R^{* r} R R^{* r} L\left(R^{* r} R\right)^{q-4} R^{* r} R R^{* r} C, \\
& \underline{P}_{s}^{\prime}=R^{* r} R R^{* r} L\left(R^{* r} R\right)^{q-4} R^{* r} R R^{* r} L .
\end{aligned}
$$

From Remark 5.4 and since $r$ is even, it follows that $\underline{P}_{s}^{c}$ is odd. So, from the expression of $\underline{P}_{a(s)}$ we obtain $\underline{P}_{s}^{c}<\underline{P}_{a(s)}^{\infty}$ and $\left(\underline{P}_{s}^{\prime}\right)^{\infty}<\underline{P}_{a(s)}^{\infty}$. By Theorem II.2.8 of [CE], $\underline{P}_{a(s)}^{\infty}<\underline{P}_{t}^{\infty}$, and Case A follows for $r>0$ even. Similarly we obtain Case A for $r>0$ odd. The proof of Case A for $r=0$ is the above one deleting all the $R^{* r}$.

Case B. $q=3$. We note that $r \geqslant 1$. Now we define $a(s)=2^{r-1} p$ with $p \geqslant 9$ and $t \rightarrow a(s)$.

Let $r$ be even. By Theorem II.2.9 of [CE] and Remark 5.4, we have

$$
\begin{aligned}
& \underline{P}_{a(s)}=R^{*(r-1)} L R^{*(r-1)} R\left(R^{*(r-1)} L\right)^{p-2}=R^{* r} R\left(R^{* r} L\right)^{3}\left(R^{*(r-1)} L\right)^{p-8}, \\
& \underline{P}_{s}^{c}=R^{* r} R R^{* r} L R^{* r} C, \\
& \underline{P}_{s}^{\prime}=R^{* r} R R^{* r} L R^{* r} L .
\end{aligned}
$$

Now Case B follows similarly to Case A. Q.E.D.

Proposition 5.6. Let $f$ be a unimodal map and suppose $P$ is the unique MPO of $f$ and $c \in P$. Then $P$ is a $Q M M P O$.

Proof. Since $c \in P$ we have $p_{n}=b$, where $n$ is the period of $P$ and $P=$ $\left\{p_{1}, p_{2}, \ldots, p_{n}\right\}$ with $p_{1}<p_{2}<\cdots<p_{n}$. Then we have $\underline{I}\left(p_{n}\right)=R \cdots C=\underline{D} C$ and $|\underline{D}|=n-1$. Now we consider two cases:

Case A. $n=2^{m} q$ with $m \geqslant 0$ and $q \geqslant 3$ odd. If $\underline{I}\left(p_{n}\right)=\underline{P}_{n}^{c}$ we are finished. Then we suppose $\underline{I}\left(p_{n}\right) \neq \underline{P}_{n}^{c}$. Now we claim $\underline{P}_{n}^{\infty}<<\underline{I}(b)=\underline{I}\left(p_{n}\right)$.

To prove the claim we must check either $S^{k}\left(\underline{P}_{n}^{\infty}\right)<(\underline{D} L)^{\infty}$ if $\underline{D}$ is even or $S^{k}\left(\underline{P}_{n}^{\infty}\right)<(\underline{D} R)^{\infty}$ if $\underline{D}$ is odd, for all $k \geqslant 0$. But, since $\underline{P}_{n}^{\infty}$ is maximal we have $S^{k}\left(\underline{P}_{n}^{\infty}\right)<\underline{P}_{n}^{\infty}$ for all $k \geqslant 0$. Then it is enough to show that either $\underline{P}_{n}^{\infty}<(\underline{D} L)^{\infty}$ if $\underline{D}$ is even or $\underline{P}_{n}^{\infty}<(\underline{D} R)^{\infty}$ if $\underline{D}$ is odd. We consider the case $\underline{D}$ even. The proof for the case $\underline{D}$ odd follows similarly.

Suppose $(\underline{D} L)^{\infty}$ is a periodic sequence of period $k$. Then $k$ is either $n$ or $n / 2$. To prove this we set $p_{s}=c$ and take a unimodal map $g$ on $\left[a^{\prime}, b^{\prime}\right]$ such that:

(i) $b^{\prime}>b$ and $a^{\prime} \leqslant a$;

(ii) $g$ coincides with $f$ restricted to $\left[a, p_{s}\right]$ and $\left[p_{s+1}, b\right]$;

(iii) the maximum of $g, c^{\prime} \in\left(p_{s}, p_{s+1}\right)$.

Then, since $\underline{I}_{f}\left(p_{n}\right)=\underline{D} C$ we have $\underline{I}_{g}\left(p_{n}\right)=(\underline{D} L)^{\infty}$. By Lemma II.3.2 of [CE], we have that $(\underline{D} L)^{\infty}$ is either periodic of period $n$ or periodic of period $n / 2$ because $P$ is a periodic orbit of $g$ of period $n$.

Now, we have $(\underline{D} L)^{\infty}=\underline{A}^{\infty}$ for some $\underline{A}$ with $|\underline{A}|=k$ and either $k \rightarrow n$ or $k=n$. Since $\underline{I}(b)=\underline{I}\left(p_{n}\right)=\underline{D} C$ and $L^{\infty}$ is maximal, by Corollary II.2.4 of $[\mathrm{CE}](\underline{D} L)^{\infty}=$ $\underline{D} * L^{\infty}$ is maximal. So $\underline{A}^{\infty}$ is maximal. Hence, by the definition of min-max and Theorem II.2.8 of [CE] we have $\underline{P}_{n}^{\infty} \leqslant \underline{P}_{k}^{\infty} \leqslant \underline{A}^{\infty}=(\underline{D} L)^{\infty}$.

If $\underline{P}_{n}^{\infty}=(\underline{D} L)^{\infty}$ we have $\underline{I}\left(p_{n}\right)=\underline{D} C=\underline{P}_{n}^{c}$, which is impossible. Therefore $\underline{P}_{n}^{\infty}<$ $(\underline{D} L)^{\infty}$ and this proves the claim. 
Since $I(a)<\underline{P}_{n}^{\infty}$, by Theorem II.3.8 of [CE] there is an $x \in[a, b]$ such that $\underline{I}(x)=\underline{P}_{n}^{\infty}$. By Lemma II.3.4 of [CE] there is a periodic point $z$ of period $n$ such that either $\underline{I}(z)=\underline{P}_{n}^{\infty}$ or $\underline{I}(z)=\underline{P}_{n}^{c}$. Since $\underline{D} C=\underline{I}\left(p_{n}\right)$ is different from $\underline{P}_{n}^{\infty}$ and $\underline{P}_{n}^{c}$ we have $z \neq p_{n}$. Moreover, if $\underline{I}(z)=\underline{P}_{n}^{\infty}$, then $c \notin \operatorname{Orb}(z)$. So $\operatorname{Orb}(z) \neq P$ and this is a contradiction with the uniqueness of the MPO. If $\underline{I}(z)=\underline{P}_{n}^{c}$ then $f^{n}(z)=c=f^{n}\left(p_{n}\right)$ and, since $z \neq p_{n}$, this is not possible.

Case B. $n=2^{m}$ with $m \geqslant 1$. We consider the case $\underline{D}$ even. If $\underline{D}$ is odd the proof is analogous.

We take the sequence $(\underline{D} L)^{\infty}$. As in Case $\mathrm{A}(\underline{D} L)^{\infty}$ is periodic of period either $n$ or $n / 2$. Now we consider two subcases.

Case B.1. $(\underline{D} L)^{\infty}$ is a periodic sequence of period $n$. By Theorem II.2.9 of [CE] and Remark 5.4, we have $\underline{P}_{2^{k}}=R^{* k} * R$ is odd for every $k \geqslant 1$. Therefore, since $\underline{D} L$ is even, $(\underline{D} L)^{\infty}$ is different from $\left(R^{* m} * R\right)^{\infty}$. Moreover, because $(\underline{D} L)^{\infty}$ is periodic of period $n$ we have that $(\underline{D} L)^{\infty}$ is different from $\left(R^{* k} * R\right)^{\infty}$ with $k \geqslant 1$ and $k \neq m$. On the other hand, we note that $(\underline{D} L)^{\infty}=(R \cdots)^{\infty}$ is maximal and $R^{\infty}$ is the smallest maximal sequence starting with $R$. Hence, by Lemma II.2.12 of [CE] we obtain that $\left(R^{* k} * R\right)^{\infty}<(\underline{D} L)^{\infty}$ for every $k \geqslant 1$. So, $\underline{P}_{2^{k}}^{\infty}<<\underline{I}(b)$ for every $k \geqslant 1$.

Obviously $\underline{I}(a)<\underline{P}_{2^{k}}^{\infty}$ for every $k \geqslant 1$. Therefore, taking $k=m+1$, and by Theorem II.3.8 and Lemma II.3.4 of [CE], there is a periodic point of period $2^{m+1}$, but this is not possible because $P$ is a MPO. Thus, Case B.1 is not possible.

Case B.2. $(\underline{D} L)^{\infty}$ is a periodic sequence of period $n / 2$. Then $(\underline{D} L)^{\infty}=\underline{A}^{\infty}$ with $|\underline{A}|=2^{m-1}$. If $\underline{A}$ is different from $R^{*(m-1)} * R$, as in Case B.1, we arrive at a contradiction. Thus, $\underline{A}=R^{*(m-1)} * R$. Then, since $\left(R^{*(m-1)} * R\right)^{2}=\underline{A}^{2}=\underline{D} L$, we have that $R^{*(m-1)} * R$ must be equal to $R^{*(m-1)} L$. Therefore $R^{*(m-1)}$ is odd and $\underline{D}=R^{*(m-1)} L R^{*(m-1)}$. Hence $\underline{D}=R^{* m}$ and $\underline{D}$ is even. By Theorem II.2.9 of [CE] we have $\underline{P}_{n}=\underline{P}_{2^{m}}=R^{* m} * R=R^{* m} R=\underline{D} R$. Then $\underline{I}\left(p_{n}\right)=\underline{D} C=\underline{P}_{n}^{c}$ and this finishes the proof of Proposition 5.6. Q.E.D.

LEMMA 5.7. Let $f$ be a unimodal map and $P=\left\{p_{1}, p_{2}, \ldots, p_{n}\right\}$ a QMMPO of period $n$ with $p_{1}<p_{2}<\cdots<p_{n}$. Let $\bar{f}$ be the P-linearization of $f$ restricted to $\left[p_{1}, p_{n}\right]$. Then $P$ is a MPO of $\bar{f}$.

Proof. We have that the itinerary of $p_{n}$ with respect to $\bar{f}$ is $\underline{I}_{f}^{-}\left(p_{n}\right)=\underline{P}_{n}^{c}$. Suppose $P$ is not a MPO of $\bar{f}$. That is, there exists $t \in P(\bar{f})$ such that $t \rightarrow n$. Now we consider two cases.

Case A. $t=2^{m}$ for some $m \geqslant 1$.

Case B. $t=2^{m} q$ where $m \geqslant 0$ and $q \geqslant 3$ odd.

In Case A, clearly we have $n=2^{k}$ with $0<k<m, k \in N$. Let $Q=\left\{q_{1}, q_{2}, \ldots, q_{t}\right\}$ with $q_{1}<q_{2}<\cdots<q_{t}$ a MPO of $\bar{f}$. By Lemma II.3.2 of [CE], we have that $\underline{I}_{\bar{f}}\left(q_{t}\right)$ is periodic of period either $2^{m}$ or $2^{m-1}$. Hence, by Theorem II.2.8 of [CE], the definition of min-max and the maximality of $\underline{I}_{f}^{-}\left(q_{t}\right)$, we have $\underline{P}_{n}^{\infty} \leqslant \underline{P}_{2^{m-1}}^{\infty} \leqslant \underline{I}_{f}\left(q_{t}\right)$. Moreover, by Lemma 5.5, $\underline{P}_{n}^{c}<\underline{P}_{n}^{\infty}$. Therefore, by Lemma II.1.2 of [CE], $p_{n}<q_{t}$, which is impossible.

In Case B we arrive at a contradiction similar to Case A. Q.E.D.

Proof of TheOrem 3.4. We write $P=\left\{p_{1}, p_{2}, \ldots, p_{n}\right\}$ with $p_{1}<p_{2}<\cdots<p_{n}$. 
"If part". By Lemma II.3.2 of [CE] we have that $\underline{I}_{f}\left(p_{n}\right)$ is periodic of period either $n$ or $n / 2$. Let $\bar{f}$ be the restriction to $\left[p_{1}, p_{n}\right]$ of the $P$-linearization of $f$. Obviously, $P$ is a SPO of $\bar{f}$ and $\bar{I}_{\bar{f}}\left(p_{n}\right)=\underline{D} C$ has length $n$. By Theorem 2.6(2), Proposition 2.7 and Theorem 2.17(2), we have that $P$ is the unique MPO of $\bar{f}$. Now, by Proposition 5.6, $\underline{I}_{f}\left(p_{n}\right)=\underline{P}_{n}^{c}$. Therefore $P$ is a QMMPO of $f$.

"Only if part". Let $\bar{f}$ be as above. By Lemma 5.7 we have that $P$ is a MPO of $\bar{f}$. Then, by Remark 3.3 we have that $P$ is a SPO of $\bar{f}$. Hence, by Definition $2.5, P$ is a SPO of $f$.

Now we prove the last statement of Theorem 3.4. In the proof of the "if part" we showed that for a SPO of a map $f$, we have $\underline{I}_{f}\left(p_{n}\right)=\underline{P}_{n}^{c}$. By Lemma 5.2 and Remark 5.3 we can compute explicitly the unique behavior of $\bar{f}$ (and $f$ ) on $P$ expressed in terms of the rotation sequence. This completes the proof of Theorem 3.4. Q.E.D.

Proof of Theorem 3.5. First suppose $P(f) \subset\left\{2^{m}: m \geqslant 0\right\}$. Then Theorem 2.6(3) finishes the proof for this case. Now suppose the above condition is not satisfied. Let $t$ be the minimum of $P(f)$ in Sarkovskii's ordering. Clearly $t=2^{m} q$ with $m \geqslant 0$ and $q \geqslant 3$ odd. Then, by Remark 3.3, we have that all the periodic orbits of period $t$ are simple. Thus, we must prove Theorem 3.5 for every $n \in P(f)$ with $t \rightarrow n$. By the proof of Theorem II.3.10, Theorem II.3.8 and Lemmas II.3.2 and II.3.4 of [CE], we have that there exists a periodic point $x$ of period $n$ such that either $\underline{I}(x)=\underline{P}_{n}^{\infty}$ or $\underline{I}(x)=\underline{P}_{n}^{c}$. By Theorem 3.4, we have that the orbit of $x$ is a SPO of $f$. Q.E.D.

The next proposition characterizes a SPO (QMMPO) $P$ of a unimodal map $f$ in terms of the behavior of $f$ on $P$.

Proposition 5.8. Let $f$ be a unimodal map and $P=\left\{p_{1}, p_{2}, \ldots, p_{n}\right\}$ a SPO of period $n=2^{m} q$ with $m \geqslant 0$ and $q \geqslant 1$ odd. Suppose $p_{1}<p_{2}<\cdots<p_{n}, t=$ $(q+1) / 2, w=2^{m}$ and $r_{m}=\sum_{j=2, j e v e n}^{m} 2^{m-j}$. Then the following hold.

(1) There exists a point $z>c$ such that $f(z)=z$ and $z<p_{t+1}$ if $m=0$ or $z<p_{(n / 2)+1}$ if $m \geqslant 1$.

(2) If $m=0$ then $P$ is a SPO ${ }^{+}, p_{1}<c$ and $p_{3}>c$.

(3) If $m \geqslant 1$ and $q=1$, then $p_{r_{m}}<c$ and $p_{r_{m}+2}>c$.

(4) If $m \geqslant 1$ and $q \geqslant 3$ odd, then $f \equiv R\left(Q_{k}\right)$ for $k=1,2, \ldots, r_{m}$ and $f \equiv-R\left(Q_{k}\right)$ for $k=r_{m}+2, r_{m}+3, \ldots, w$. Furthermore, $f$ on $Q_{r_{m}+1}$ satisfies (1) (resp. (3)) of Proposition 4.6 if and only if $m$ is even (resp. odd). In short, using the notation of Definition 2.9, we have that $p_{1}^{r_{m}+1}<c$ and $p_{3}^{r_{m}+1}>c\left(\right.$ resp. $p_{q-2}^{r_{m}+1}<c$ and $\left.p_{q}^{r_{m}+1}>c\right)$.

(5) If $m \geqslant 1$ and $q \geqslant 3$ odd, then $Q_{i}$ is a SPO ${ }^{+}$of $f^{w}$ of period $q$ if and only if $i$ is even. Moreover, $Q_{r_{m}+1}$ is a SPO ${ }^{+}$of $f^{w}$ of period $q$ if and only if $m$ is even.

Proof. (2) If $P$ is a SPO ${ }^{-}$then, by Definition 2.3, we have $f\left(p_{t}\right)<P_{t}$ and $p_{t}=f\left(p_{q}\right)<p_{q}$. Then $z<p_{t}, p_{q}$ where $z$ is the unique fixed point of $f$ on the right of $c$. Since $f$ is order reversing on $[c, b]$ we have $f\left(p_{q}\right)<f\left(p_{t}\right)$, which is a contradiction. Hence $P$ is a SPO ${ }^{+}$. Then, since $f\left(p_{2}\right)=p_{q}$, we have $p_{1}<c$ and $p_{3}>c$.

(1) From (2), Definitions 2.3 and 2.8 and Remark 3.3 we have $f\left(p_{i}\right)<p_{i}$ if and only if either $i \geqslant t+1$ and $m=0$ or $i \geqslant(n / 2)+1$ and $m \geqslant 1$. Therefore (1) follows. 
(3) Let $\bar{f}$ be the $P$-linearization of $f$ restricted to $\left[p_{1}, p_{n}\right]$. Then, from Theorem 3.4 we have $\underline{I}_{f}\left(p_{n}\right)=\underline{P}_{n}^{c}$. To prove (3) it is sufficient to show that $\underline{P}_{n}^{c}$ has exactly $r_{m}$ symbols $L$. We prove this by induction with respect to $m$. If $m=1$ we have $\underline{P}_{2}^{c}=R C$ and $r_{1}=0$. For $\underline{P}_{4}^{c}=R L R C$ we have $r_{2}=1$. Now suppose this is true for $n=2^{m}$. To prove this is true for $2 n=2^{m+1}$, we suppose $m$ even. The case $m$ odd follows similarly.

By Theorem II.2.9 of [CE], $\underline{P}_{n}^{c}=R^{* m} C$ and, by Remark 5.4, $R^{* m}$ is even. Since $\underline{P}_{2 n}^{c}=R^{*(m+1)} C$ and $R^{*(m+1)}=R^{* m} R R^{* m}$, we have that the number of $L$ 's in $\underline{P}_{2 n}^{c}$ is $2 r_{m}$. Then, since $m$ is even,

$$
r_{m+1}=\sum_{\substack{j=2 \\ j \text { even }}}^{m+1} 2^{m+1-j}=\sum_{\substack{j=2 \\ j \text { even }}}^{m} 2 \cdot 2^{m-j}=2 r_{m} .
$$

Hence, the number of $L$ 's in $\underline{P}_{2 n}^{c}$ is $r_{m+1}$ and this finishes the proof of (3).

(4) We take a map $g$ as in the proof of Lemma 4.14. Clearly, $g$ is a unimodal map. Then by (3) we have $q_{r_{m}+1}=c$. Therefore, $c \in G_{r_{m}+1}$ and this implies $f \equiv R\left(Q_{i}\right)$ for $i=1,2, \ldots, r_{m}$ and $f \equiv-R\left(Q_{i}\right)$ for $i=r_{m}+2, \ldots, w$.

In order to prove the second statement of (4) we claim that $r_{m}$ is odd if and only if $m$ is even. Since we proved in (3) that $r_{m+1}=2 r_{m}$ if $m$ is even, we have that if $m$ is odd, then $r_{m}$ is even. Moreover, if $m$ is even

$$
r_{m}=\sum_{\substack{j=2 \\ j \text { even }}}^{m} 2^{m-j}=2^{0}+\sum_{\substack{j=2 \\ j \text { even }}}^{m-1} 2^{m-j}=1+2\left(\sum_{\substack{j=2 \\ j \text { even }}}^{m-1} 2^{m-j-1}\right)
$$

Then $r_{m}$ is odd and this proves the claim.

Since

$$
S_{m}=\left|\left\{i: f \equiv-R\left(Q_{i}\right), i=1,2, \ldots, w\right\}\right|=2^{m}-r_{m}-1,
$$

we have that $S_{m}$ is odd if and only if $r_{m}$ is even. Hence, by the claim, $S_{m}$ is even if and only if $m$ is even. Then by Proposition 4.6(5) $f$ satisfies either (1) or (2) of Proposition 4.6 on $Q_{r_{m}+1}$ if and only if $m$ is even. Moreover, since $f$ is unimodal, we have that $f$ satisfies Proposition 4.6(1) on $Q_{r_{m}+1}$ if and only if $m$ is even. Similarly we have that $f$ satisfies Proposition 4.6(3) if and only if $m$ is odd.

(5) Again we take an unimodal map $g$ as in the proof of Lemma 4.14, with $Q=\left\{q_{1}, q_{2}, \ldots, q_{w}\right\}$ a SPO of $g$. We note that $\underline{I}_{g}\left(q_{w}\right)$ is in some sense the " $\underline{I}_{f}\left(Q_{w}\right)$ ". Let $\underline{I}_{g}\left(q_{w}\right)=I_{0} I_{1} \cdots I_{w-1}$, and for every $r \in\{1,2, \ldots, w-1\}$ choose $i(r)$ such that $g^{i(r)}\left(q_{w}\right)=q_{r}$.

Then to prove (5) we shall show the following statements:

(a) $r$ is even if and only if $I_{0} I_{1} \cdots I_{i(r)-1}$ is even.

(b) $Q_{w}$ is a $\mathrm{SPO}^{+}$of $f^{w}$. Moreover, $Q_{r}$ is a $\mathrm{SPO}^{+}$of $f^{w}$ if and only if $I_{0} I_{1} \cdots I_{i(r)-1}$ is even.

Clearly, from (a) and (b) the first statement of Proposition 5.8(5) follows.

To prove (a) we define $T_{j}=\left\{q_{2 j-1}, q_{2 j}\right\}$ for $j=1,2, \ldots, w / 2$. We note that $T=\left\{T_{j}: j=1,2, \ldots, w / 2\right\}$ is a simple set because $Q$ is a SPO. We take either 
$j=r / 2$ if $r$ is even or $j=(r+1) / 2$ if $r$ is odd. Then, since $g^{i(r)}\left(q_{w}\right) \in T_{j}$, we have

$$
g^{i(r)}\left(T_{w / 2}\right)=T_{j}
$$

(because $T$ is a simple set).

Now suppose $I_{0} I_{1} \cdots I_{i(r)-1}$ even. We shall prove that $r$ is even. Consider the orbit of $T_{w / 2}$ by $g$ :

$$
\left\{T_{w / 2}, g\left(T_{w / 2}\right), g^{2}\left(T_{w / 2}\right), \ldots, g^{i(r)}\left(T_{w / 2}\right)\right\} .
$$

From (*) we have that $g$ passes an even number of times through the decreasing part of the graph of $g$. Since $g$ is unimodal $g^{i(r)}$ is order preserving on $T_{w / 2}$. Then, from (*) we have $q_{r}=g^{i(r)}\left(q_{w}\right)=q_{2 j}$. Then $r$ is even. Similarly, we have that if $I_{0} I_{1} \cdots$ $I_{i(r)-1}$ is odd, then $r$ is odd. Therefore, (a) follows.

To prove (b), first suppose, as above, that $I_{0} I_{1} \cdots I_{i(r)-1}$ is even. From the fact that $f$ behaves on $\left\{Q_{i}: i=1,2, \ldots, w\right\}$ as $g$ on $Q$ and $f$ is unimodal, we have that $\left|S_{r}\right|$ is even (here we use the notation of Proposition 4.6(6)). Then, from Proposition 4.6(a) and (4), we have that $Q_{r}$ is a $\mathrm{SPO}^{+}$of $f^{w}$. It follows similarly that $Q_{r}$ is a $\mathrm{SPO}^{-}$for $f^{w}$ if $I_{0} I_{1} \cdots I_{i(r)-1}$ is odd.

On the other hand, since $f\left(Q_{r_{m}+1}\right)=Q_{w}$, we have $S_{w}=\varnothing$ and $\left|S_{w}\right|=0$, even. Again by Proposition 4.6(a) and (4), $Q_{w}$ is an $\mathrm{SPO}^{+}$for $f^{w}$.

From the proof of (4) we have that $r_{m}$ is odd if and only if $m$ is even. This completes the proof of (5). Q.E.D.

ACKNOWLedgements. We thank Jaume Gallifa and Enric Ribot for their suggestions for elaborating the computer program which enabled us to obtain the definition of the simple periodic orbit. Also, we are indebted to the referee, whose suggestions improved the exposition of the paper.

ADDED IN PROOF. Recently the authors received the Chung-wu Ho preprint entitled On the structure of the minimum orbits of periodic points for maps of the real line. In it a different proof of Theorem 2.17 is given.

\section{REFERENCES}

[AS] Ll. Alseda and R. Serra, The simple periodic orbits in the unimodal maps, Proc. Dynamical Systems and Chaos (Sitges, 1982), Lecture Notes in Phys. 179, Springer, Berlin, 1982, pp. 248-249.

[B11] L. Block, Simple periodic orbits of mappings of the interval, Trans. Amer. Math. Soc. 254 (1979), 391-398.

[B12] _ Periods of periodic points of maps of the circle which have a fixed point, Proc. Amer. Math. Soc. 82 (1981), 481-486.

[BGMY] L. Block et al, Periodic points and topological entropy of one dimensional maps, Lecture Notes in Math., vol. 819, Springer-Verlag, 1980, pp. 18-34.

[CE] P. Collet and J. P. Eckmann, Iterated maps on the interval as dynamical systems, Birkhäuser,Basel, 1980.

[G] C. Gillot, Characterisation des suites de rotation d' un endomorphisme unimodal de [0,1], C. R. Acad. Sci. Paris 293 (1981), 249-251.

[GM] I. Gumowski and C. Mira, Dynamique chaotique, Cepadues ed., Toulouse, 1980.

[L] J. Llibre, On the rotation sequences of the unimodal maps, Barcelona, 1982 (preprint).

[LIR] J. Llibre and A. Reventos, On the structure of the set of periodic points of a continuous map of the interval with finitely many periodic points, Arch. Math. 39 (1982), 331-334.

[Sa] A. N. Sarkovskii, Coexistence of cycles of a continuous map of a line into itself, Ukrain. Mat. Zh. 16 (1964), 61-71. 
[St] P. Stefan, A theorem of Sarkouskii on the existence of periodic orbits of continuous endomorphisms of the real line, Comm. Math. Phys. 54 (1977), 237-248.

Departament de T. Econòmca, Facultad de Ciències Econòmoues, Universitat Autònoma de Barcelona, Bellaterra, Barcelona, Spain

Secció de Matemitioues, facultad de Ciències, Universitat Autónoma de Barcelona, Bellaterra, Barcelona, Spain

I. N. B. Sant Cugat, Sant Cugat del Vallès, Barcelona, Spain 